\title{
The 3-D shaping of NGC 6741: A massive, fast-evolving Planetary Nebula at the recombination-reionization edge ${ }^{\star, \star \star}$
}

\author{
F. Sabbadin ${ }^{1}$, S. Benetti ${ }^{1}$, E. Cappellaro ${ }^{2}$, R. Ragazzoni ${ }^{3}$, and M. Turatto ${ }^{1}$ \\ 1 INAF - Osservatorio Astronomico di Padova, vicolo dell'Osservatorio 5, 35122 Padova, Italy \\ e-mail: sabbadin@pd.astro.it \\ 2 INAF - Osservatorio Astronomico di Capodimonte, via Moiariello 11, 80131 Napoli, Italy \\ 3 INAF - Osservatorio Astrofisico di Arcetri, Largo E. Fermi 5, 50125, Italy
}

Received 29 November 2004 / Accepted 17 January 2005

\begin{abstract}
We infer the gas kinematics, diagnostics and ionic radial profiles, distance and central star parameters, nebular photo-ionization model, spatial structure and evolutionary phase of the Planetary Nebula NGC 6741 by means of long-slit ESO NTT+EMMI high-resolution spectra at nine position angles, reduced and analysed according to the tomographic and 3-D methodologies developed at the Astronomical Observatory of Padua (Italy).

NGC 6741 (distance $\simeq 2.0 \mathrm{kpc}$, age $\simeq 1400 \mathrm{yr}$, ionized mass $\left.M_{\mathrm{ion}} \simeq 0.06 M_{\odot}\right)$ is a dense (electron density up to $12000 \mathrm{~cm}^{-3}$ ), high-excitation, almost-prolate ellipsoid $(0.036 \mathrm{pc} \times 0.020 \mathrm{pc} \times 0.018 \mathrm{pc}$, major, intermediate and minor semi-axes, respectively), surrounded by a sharp low-excitation skin (the ionization front), and embedded in a spherical (radius $\simeq 0.080 \mathrm{pc}$ ), almostneutral, high-density $\left(n(\mathrm{HI}) \simeq 7 \times 10^{3}\right.$ atoms $\left.\mathrm{cm}^{-3}\right)$ halo containing a large fraction of the nebular mass $\left(M_{\text {halo }} \geq 0.20 M_{\odot}\right)$. The kinematics, physical conditions and ionic structure indicate that NGC 6741 is in a deep recombination phase, started about 200 years ago, and caused by the rapid luminosity drop of the massive $\left(M_{*}=0.66-0.68 M_{\odot}\right)$, hot $\left(\log T_{*} \simeq 5.23\right)$ and faint $\left(\log L_{*} / L_{\odot} \simeq 2.75\right)$ post-AGB star, which has exhausted the hydrogen-shell nuclear burning and is moving along the white dwarf cooling sequence. The general expansion law of the ionized gas in NGC $6741, V_{\exp }\left(\mathrm{km} \mathrm{s}^{-1}\right)=13 \times R^{\prime \prime}$, fails in the innermost, highest-excitation layers, which move slower than expected. The observed deceleration is ascribable to the luminosity drop of the central star (the decreasing pressure of the hot-bubble no longer balances the pressure of the ionized gas), and appears in striking contrast to recent reports inferring that acceleration is a common property of the Planetary Nebulae innermost layers. A detailed comparative analysis proves that the "U"-shaped expansion velocity field is a spurious, incorrect result due to a combination of: (a) simplistic assumptions (spherical shell hypothesis for the nebula); (b) unfit reduction method (emission profiles integrated along the slit); and (c) inappropriate diagnostic choice ( $\lambda 4686 \AA$ of He II, i.e. a thirteen finestructure components recombination line). Some general implications for the shaping mechanisms of Planetary Nebulae are discussed.
\end{abstract}

Key words. planetary nebulae: individual: NGC 6741- ISM: kinematics and dynamics

\section{Introduction}

The sentence by the late Professor Lawrence H. Aller (1994): "A nebula is a three-dimensional structure for which we obtain a two-dimensional projection" fully synthesizes the many, so far unsolved, observational limitations and interpretation

^ Based on observations made with: ESO Telescopes at the La Silla Observatories (program ID 65.I-0524), and the NASA/ESA Hubble Space Telescope, obtained from the data archive at the Space Telescope Institute. Observing programs: GO 7501 and GO 8773 (P. I. Arsen Hajian). STScI is operated by the association of Universities for Research in Astronomy, Inc. under the NASA contract NAS 5-26555. We extensively apply the photo-ionization code CLOUDY, developed at the Institute of Astronomy of the Cambridge University (Ferland et al. 1998).

$\star \star 7$ movies including movie of complete Fig. 18 are only available in electronic form at http://www. edpsciences.org problems connected to Planetary Nebula (PN) research, leading to: (a) rough spatio-kinematical reconstruction; (b) unrealistic assumptions for the gas parameters (in particular, electron temperature $\left(T_{\mathrm{e}}\right)$ and electron density $\left(N_{\mathrm{e}}\right)$ constant all across the nebula); and (c) proliferation of kinematical, physical and evolutional models, frequently based on the mere nebular morphology (i.e. appearance).

The tomographic and 3-D analyses developed at the Astronomical Observatory of Padua (Sabbadin et al. 2004, and references therein) have overcome the stumbling block of nebular de-projection, rebuilding the ionic spatial structure, and allow us a direct comparison of each real, true PN with the current theoretical evolutionary models (Schönberner et al. 1997; Steffen et al. 1998; Marigo et al. 2001), the detailed hydrodynamical simulations (Icke et al. 1992; Frank 1994; Mellema 1997; Perinotto et al. 2004a) and the updated photo-ionization codes (Ferland et al. 1998; Ercolano et al. 2003). 
Though the observational starting point is common - i.e. long-slit spectra -, the "philosophy" of tomography is just opposite of the conventional method. The latter compacts the spectrum along the slit (in practice, it restricts the nebula to a point), and gives mean, integrated results (line flux, expansion velocity, $T_{\mathrm{e}}, N_{\mathrm{e}}$, ionization etc.). Vice versa, tomography is based on a pixel-to-pixel analysis of both flux and velocity, and furnishes the bi-dimensional structure (in different ions) of the radial slice of the nebula intercepted by the spectrograph slit. Later on, a 3-D rendering procedure combines all tomographic slices and provides the true spatial distribution of the kinematics, physical conditions $\left(T_{\mathrm{e}}\right.$ and $\left.N_{\mathrm{e}}\right)$ and ionic and chemical abundances with unprecedented accuracy. Tomography needs spectra at high "relative" spatial (SS) and spectral (RR) resolutions ( $\mathrm{SS}=r / \Delta r, r=$ apparent radius, $\Delta r=$ seeing; $\mathrm{RR}=V_{\exp } / \Delta V, \Delta V=$ instrument spectral resolution). It is based on the simple consideration that the position, depth and density of each elementary volume within an extended, regularly expanding nebula can be, in principle, derived from the radial velocity, $F W H M$ and flux, respectively, of the corresponding emission.

So far we have studied NGC 40 (Sabbadin et al. 2000a), NGC 1501 (Sabbadin et al. 2000b; Ragazzoni et al. 2001), NGC 6565 (Turatto et al. 2002), NGC 6818 (Benetti et al. 2003) and NGC 7009 (Sabbadin et al. 2004). Here we present the results for NGC 6741.

NGC 6741 (PN G033.8-02.6, Acker et al. 1992) is a compact $\left(\right.$ main body $\simeq 7^{\prime \prime} \times 5^{\prime \prime}$, halo diameter $\simeq 15^{\prime \prime}$; Curtis 1918 ; Schwarz et al. 1992), high-surface brightness, high-excitation (class 8, Hyung \& Aller 1997) PN with a large degree of stratification of the radiation. The powering star is very hot $\left(\log T_{*} \geq 5.22\right.$, Pottasch 1981; Pottasch \& Preite-Martinez 1983; Heap et al. 1989; Tylenda et al. 1989; Kaler \& Jacoby $1989)$ and faint ( $m_{V} \simeq 19.5$; Pottasch 1981; Tylenda et al. 1989; Kaler \& Jacoby 1989; Hyung \& Aller 1997).

The [O III] and [N II] apparent morphology of NGC 6741 (sometimes called "Phantom Streak Nebula") is shown in Figs. 1 and 2 (HST-WFPC2 frames retrieved from NASA public archives). Note the vertical (equatorial?) inhomogeneous strip of absorbing knots in the [O III] image of Fig. 1, and in Fig. 2 the roundish halo and the series of weak, radial [O III] rays penetrating into the [N II] skin along and close to the apparent major axis. A multi-color HST reproduction of the nebula is given by Hajian \& Terzian at http://ad.usno.navy.mil/pne/gallery.html

The optical appearance of NGC 6741, the co-existence of ionic species with a large range of ionization potential, IP (from [O I]IP = $0 \mathrm{eV}$ to [Ne V]IP = 97.1 eV; Aller et al. 1985; Hyung \& Aller 1997) and the characteristics of the central star (hot and faint) are suggestive of a recombining PN, i.e. the star has exhausted the hydrogen-shell nuclear burning and is rapidly fading in luminosity (and temperature); the UV flux being unable to fully ionize the gas, the outer nebular regions recombine, producing the faint halo (Tylenda 1986; Phillips 2000). In many respects NGC 6741 is very much like NGC 6565 (Turatto et al. 2002).

To deepen our knowledge of the kinematics, physical conditions, ionic and spatial structure, distance and evolutionary

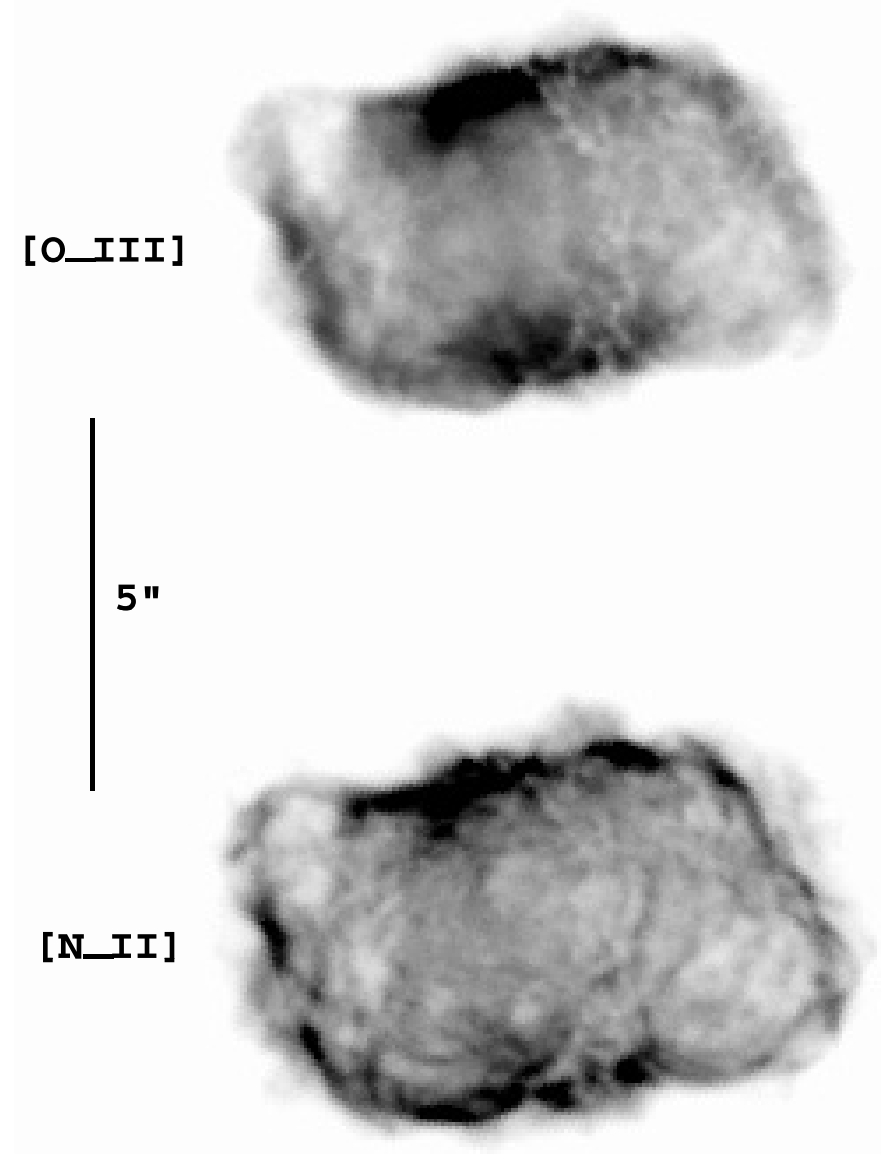

Fig. 1. Apparent morphology of NGC 6741 in the medium-excitation line $\lambda 5007 \AA$ of [O III] (upper panel), and in the low-excitation line $\lambda 6584 \AA$ of [N II] (lower panel). North is up and East to the left. HST-WFPC2 frames; programme GO 8773, P. I. Arsen Hajian.

status of NGC 6741, we have secured long-slit ESO NTT+EMMI echellograms (spectral range $\lambda \lambda 3900-7900 \AA$, spectral resolution $\lambda / \Delta \lambda=R=60000)$ at nine position angles (PA). The spectra were reduced and analysed using our 3 -D reconstruction technique (Turatto et al. 2002; Benetti et al. 2003).

Section 2 illustrates the observational procedure and the reduction method, Sect. 3 defines the kinematics of the ionized gas, Sect. 4 quantifies the interstellar and circum-nebular absorption, Sect. 5 provides the nebular distance, size and age, in Sect. 6 we discuss the parameters of the central star (temperature, luminosity, mass and evolutionary phase) and in Sect. 7 the nebular parameters $\left(T_{\mathrm{e}}, N_{\mathrm{e}}\right.$, ionic mass and structure, chemical abundances, photo-ionization model), Sect. 8 re-builds the 3-D spatio-kinematical structure, Sect. 9 contains the general discussion and Sect. 10 provides some conclusions.

\section{Observations and reductions}

NGC 6741 was observed with ESO NTT + EMMI (echelle mode; grating \#14, grism \#3) at nine equally spaced PA, under photometric sky conditions and seeing ranging between $0.50^{\prime \prime}$ and $0.70^{\prime \prime}$. The spectrograph slit (1.0" wide and $30^{\prime \prime}$ long) was centered on the nebular image, the exciting star being invisible 


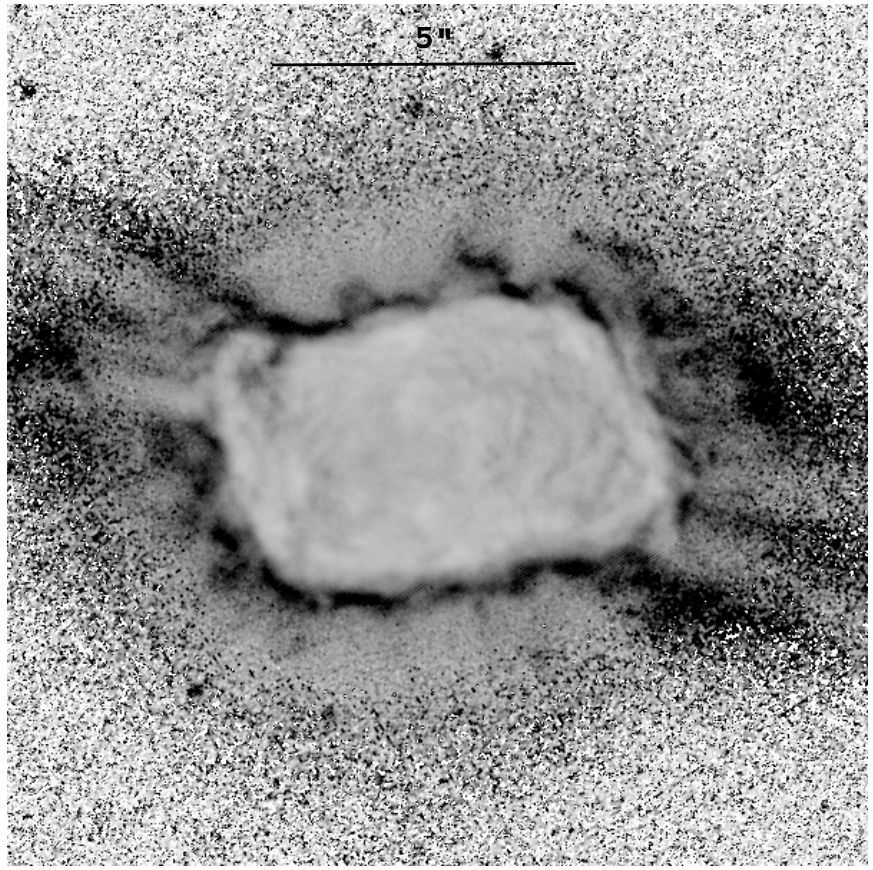

Fig. 2. Apparent $I([\mathrm{~N} \mathrm{III}]) / I([\mathrm{O} I I I])$ distribution over NGC 6741 (same WFPC2 images as Fig. 1). The sharp, inhomogeneous layer at lowexcitation ([N II]) framing the main nebula is punched, along and close to the apparent major axis, by a series of radial [O III] rays or jets penetrating into the faint, roundish halo.

in the slit-viewer. The echellograms (exposure time $600 \mathrm{~s}$ ) cover the spectral range $\lambda \lambda 900-7900 \AA$ with resolution $R \simeq$ 60000 , and provide the kinematical structure of the main ionic species within the nebular slices covered by the slit. Bias, zeroorder flat field and distortion corrections and wavelength and flux calibrations were performed according to the straightforward procedure fully described by Turatto et al. (2002).

We stress the fundamental difference between our frames, covering 80 echelle orders, and the observing procedure usually adopted for extended objects, inserting an interference filter to isolate a single order. The same is valid for the reduction and investigation methods: so far long-slit echellograms have been used to obtain, either the kinematics in a few ions (in general, [O III], H I and [N II]), or the "average" nebular properties (physical conditions, ionic and chemical abundances) integrated over the whole slit length. On the contrary, we perform a detailed, pixel-to-pixel, flux and velocity determination for a number of nebular lines, thus inferring the spatial distribution of the kinematics, diagnostics, ionic and total abundances at the same time.

The richness of physical information contained in the echellograms is illustrated in Figs. 3 and 4, presenting the spectral structure of twelve ionic species (from [OI], IP $=0 \mathrm{eV}$ to $[\mathrm{ArV}], \mathrm{IP}=59.8 \mathrm{eV}$ ) at $\mathrm{PA}=15^{\circ}$ and $75^{\circ}$ (close to the apparent minor and major axes of NGC 6741, respectively).

These figures show the spectral characteristics common at all PA, in particular: (a) large stratification of both the radiation and kinematics (compare, e.g., the ionic sequences $[\mathrm{OI}]-[\mathrm{OII}]-[\mathrm{O} \mathrm{III}]$ and $[\mathrm{Ar} \mathrm{III}]-[\mathrm{Ar} \mathrm{IV}]-[\mathrm{Ar} \mathrm{V}]$ ); and (b) blurred appearance of recombination lines (of H I, He I
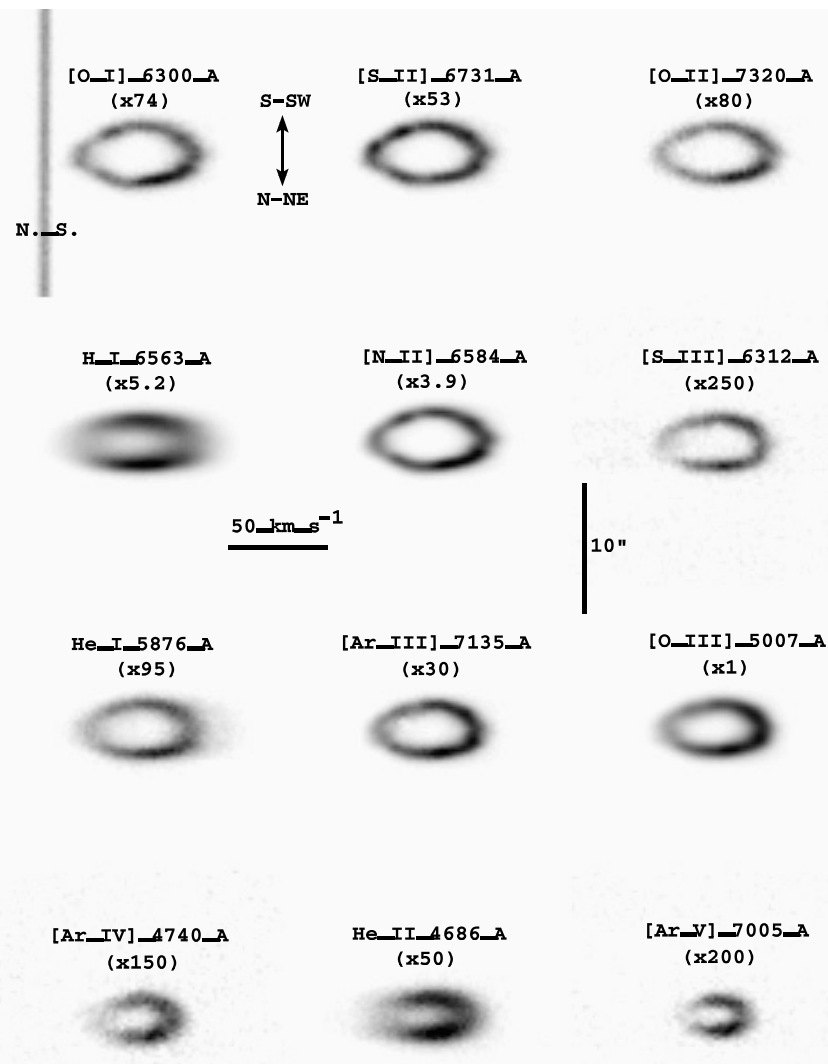

Fig. 3. Detailed spectral image of twelve ionic species in NGC 6741 at $\mathrm{PA}=15^{\circ}$ (close to the apparent minor axis), arranged in order of increasing IP (top-left to bottom-right). The original fluxes are multiplied by the factor given in parenthesis, to make each emission comparable with $\lambda 5007 \AA$ of [O III] . The blue-shifted gas is to the left. The top-left frame also shows the [O I] night-sky emission at $\lambda 6300.304 \AA$. In the top-right frame the [O II] line at $\lambda 7319 \AA$, partially blended with $\lambda 7320 \AA$, has been suppressed (for details, see the text and Fig. 5).

and He II), due to a combination of thermal motions, finestructure and expansion velocity gradient. At the same time, they highlight the kinematical and physical differences at the two PA:

- un-tilted, quite uniform emission close to the apparent minor axis (Fig. 3), although the blue-shifted gas is systematically fainter than the red-shifted gas;

- tilted, inhomogeneous lines close to the apparent major axis (Fig. 4), suggestive of a dense equatorial torus and two extended and faint polar lobes.

In the original NTT+EMMI frames, $\lambda 7320.121 \AA$ of [O II] (top-right panel in Figs. 3 and 4; hereafter $\lambda 7320 \AA$ ) is partially blended with $\lambda 7319.044 \AA$, the bluest component of the [O II] red quartet (hereafter $\lambda 7319 \AA$ ). Since the intensity ratio $I(\lambda 7320 \AA) / I(\lambda 7319 \AA)=$ constant $=3.071$ in the $N_{\mathrm{e}}$ range considered here (De Robertis et al. 1985; Keenan et al. 1999; Sharpee et al. 2004), the $\lambda 7319 \AA$ suppression was obtained in the simple fashion illustrated in Fig. 5. 


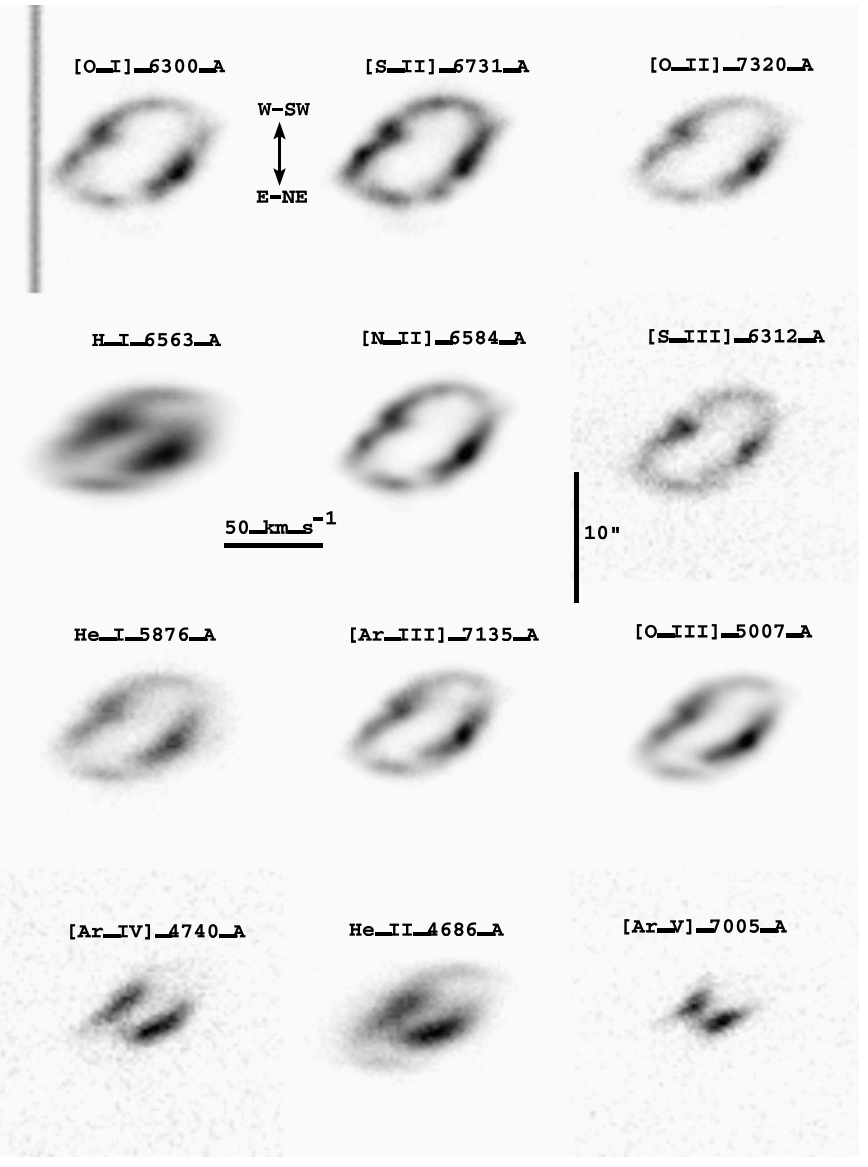

Fig. 4. Same as Fig. 3, but for $\mathrm{PA}=75^{\circ}$ (close to the apparent major axis of NGC 6741).

The "relative" spectral and spatial resolutions of our echellograms are similar to each other: $\mathrm{SS}=V_{\exp } / \Delta V \simeq 3$ to $5 \simeq \mathrm{RR}=r / \Delta r$. This means that the spatial information of NGC 6741 is as accurate as the kinematical information, and both are close to the lower limit for tomographic analysis (Ragazzoni et al. 2001). Thus, the spectral images in Figs. 3 and 4 are affected by non-negligible blurring agents.

Seeing and guiding are broadening components along the spatial (i.e. vertical) axis; in our case the full-width at half

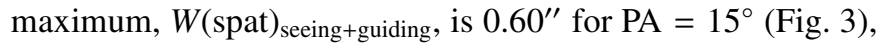
and $0.65^{\prime \prime}$ for $\mathrm{PA}=75^{\circ}$ (Fig. 4). The true, "intrinsic" profile of the nebula has:

$W(\text { spat })_{\text {intrinsic }}^{2}=W(\text { spat })_{\text {obs }}^{2}-W(\text { spat })_{\text {seeing+guiding }}^{2}$.

Concerning the velocity (i.e. horizontal) axis, we must take into account:

(1) instrumental resolution, corresponding to a Gaussian profile with $W(\text { vel })_{\text {EMMI }}=5.09 \mathrm{~km} \mathrm{~s}^{-1}$ (measured in the [O I] night-sky line at $\lambda 6300 \AA$ );

(2) thermal motions, generating a Gaussian distribution with $W(\mathrm{vel})_{\text {thermal }} \simeq 21.6 \times 10^{-2} \times T_{\mathrm{e}}^{0.5} \times m^{-0.5} \mathrm{~km} \mathrm{~s}^{-1}$, where $m$ is the atomic weight of the element (Clegg et al. 1999, and references therein); we use the radial $T_{\mathrm{e}}$ profile given in Sect. 7;

(3) turbulence, i.e. random, small-scale motions; this is a very uncertain parameter for $\mathrm{PNe}$, whose quantification is

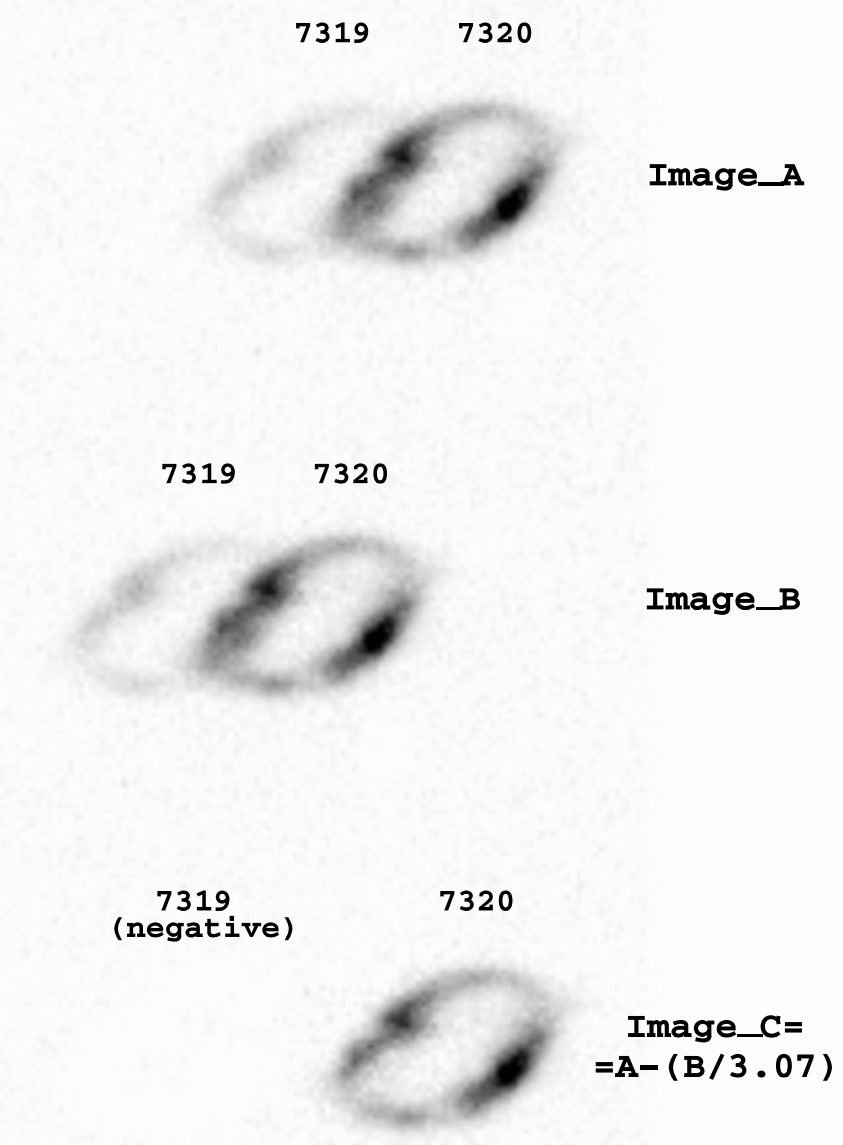

Fig. 5. De-blending procedure for the [O II] $\lambda \lambda 7319-7320 \AA$ doublet (using IRAF packages). Top panel: Image A = part of the echellogram (at PA $=75^{\circ}$ ) centered on $\lambda 7319 \AA$. Middle panel: Image $\mathrm{B}=$ part of the echellogram (at $\mathrm{PA}=75^{\circ}$ ) centered on $\lambda 7320 \AA$. Bottom panel: Image $\mathrm{C}=$ Image $\mathrm{A}-($ Image $\mathrm{B} / 3.071)$, where 3.071 corresponds to $I(7320) / I(7319)$.

deferred to a dedicated paper (in preparation), based on very-high resolution $(R \simeq 115000)$ spectra secured with the Telescopio Nazionale Galileo (TNG) + SARG on a representative sample of targets. In the present case of NGC 6741, at the moment we can only infer that $W(\text { vel })_{\text {turb }}$ is below $10.0 \mathrm{~km} \mathrm{~s}^{-1}$, as indicated by the sharpness of the spectral images of forbidden lines along the velocity (i.e. $x$ ) axis (see Figs. 3 and 4; larger turbulences should produce blurred spectral images of forbidden lines, similar to the $\mathrm{H} \alpha$ one). According to the "a posteriori" analysis presented at the end of Sect. 3, in the following we will assume $W(\mathrm{vel})_{\text {turb }}=3.5 \mathrm{~km} \mathrm{~s}^{-1}$, in partial agreement with the general results by Neiner et al. (2000), and Gesicki et al. (2003) (but see the caveat in Sect. 3);

(4) fine-structure (only for recombination lines); following Clegg et al. (1999), $W(\mathrm{vel})_{\text {fine-s. }} \simeq 7.5 \mathrm{~km} \mathrm{~s}^{-1}$ for $\mathrm{H} \alpha$; moreover, we adopt $W(\mathrm{vel})_{\text {fine-s. }}=5.0 \mathrm{~km} \mathrm{~s}^{-1}$ for $\lambda 4686 \AA$ (after suppression of the $20 \mathrm{~km} \mathrm{~s}^{-1}$ blue-shifted tail with 


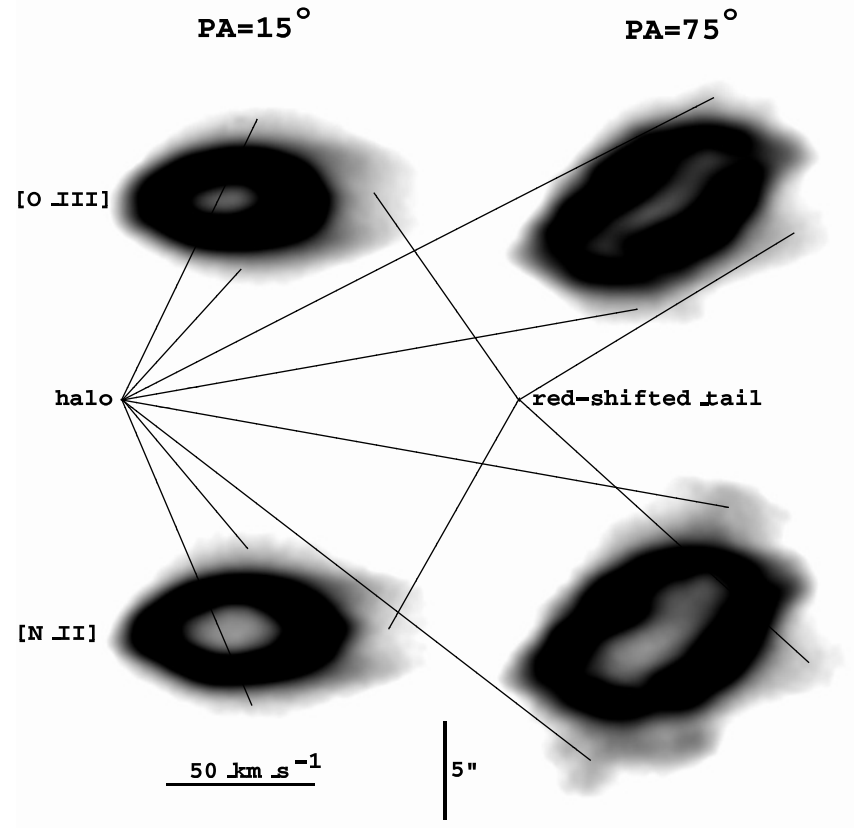

Fig. 6. High-contrast $[\mathrm{OIII}]$ and $[\mathrm{NII}]$ line profiles (in logarithmic scale) of NGC 6741 close to the apparent minor and major axes $\left(\mathrm{PA}=15^{\circ}\right.$ and $75^{\circ}$, respectively) showing the faint halo and redshifted tail. Same orientation as Figs. 3 and 4.

the method illustrated in Fig. 5) and $\lambda 6560 \AA$ of He II, and $\lambda 5876 \AA$ of He I.

Note that thermal motion and/or fine-structure represent the main broadening factors for recombination lines, whereas instrumental resolution, thermal motion and turbulence are (more or less) equivalent for forbidden lines.

The final broadening along the velocity axis is:

$W(\mathrm{vel})_{\mathrm{blur}}^{2}=\sum_{i=1}^{4} W(\mathrm{vel})_{i}^{2}$,

and the true, intrinsic full-width at half maximum:

$W(\text { vel })_{\text {intrinsic }}^{2}=W(\text { vel })_{\mathrm{obs}}^{2}-W(\text { vel })_{\text {blur }}^{2}$.

$W(\mathrm{vel})_{\text {intrinsic }}$ (in $\mathrm{km} \mathrm{s}^{-1}$ ) corresponds to the expansion velocity range of the emitting layer; it can be transformed into arcsec by means of the general expansion law of the ionized gas: in NGC 6741 we have $V_{\exp }\left(\mathrm{km} \mathrm{s}^{-1}\right)=13( \pm 1) \times R^{\prime \prime}($ see Sect. 3) and $W(\text { vel })_{\text {intrinsic }}(\operatorname{arcsec})=\left[W(\mathrm{vel})_{\text {intrinsic }}\left(\mathrm{km} \mathrm{s}^{-1}\right)\right] / 13$.

Let us consider, for example, the spectral images at PA = $15^{\circ}$ (Fig. 3). The intrinsic $F W H M$ in both the zvpc and cspl (as defined at the opening of Sect. 3) is $0.52^{\prime \prime}$ to $0.72^{\prime \prime}$ (forbidden lines), $0.77^{\prime \prime}$ to $0.95^{\prime \prime}$ (He I), $1.00^{\prime \prime}$ to $1.15^{\prime \prime}$ (He II), and $1.35^{\prime \prime}$ to $1.45^{\prime \prime}(\mathrm{HI})$, thus confirming that:

(a) each emitting region is extremely sharp;

(b) the whole spectral images must be carefully deconvolved: to this end we use the Richardson-Lucy algorithm (Richardson 1972; Lucy 1974), and a point-spread function given by a bi-dimensional Gaussian profile characterized by $W(\text { spat })_{\text {seeing+guiding }}$ and $W(\text { vel })_{\text {blur }}$.
So far we have considered the bright main shell of NGC 6741. At lower-flux cuts the faint halo appears (Fig. 6), whose spectral signature supports the recombination hypothesis (note, in particular, the broad, un-tilted halo-emission at $\mathrm{PA}=15^{\circ}$, and the broad, tilted halo-emission mimicing the kinematics of the main nebula at $\mathrm{PA}=75^{\circ}$ ). In fact, according to the current evolutionary models (e.g. Schönberner et al. 1997; Steffen et al. 1998; Marigo et al. 2001; Perinotto et al. 2004a), a PN halo can be:

- an AGB halo, i.e. the low-density envelope of an optically thin PN, directly ionized by the UV flux of the bright central star. It represents the AGB mass-loss in the pre-superwind phase, and expands at the original ejection velocity $\left(V_{\exp } \simeq\right.$ $10-15 \mathrm{~km} \mathrm{~s}^{-1}$ ), independent of the kinematical field of the main nebula;

- a recombination halo, corresponding to the outer, almost neutral parts of the main nebula, no longer reached by the UV flux of a hot central star which has exhausted the H-shell nuclear burning and passed the turnover point in stellar evolution, rapidly fading in luminosity and temperature. The recombining layers essentially retain the general kinematical properties of the main thick nebula (as observed in the halo of NGC 6741).

Figure 6 also shows the faint red-shifted tail present in the strongest emissions of NGC 6741 at all PA, whose nature (halo? interaction with the ambient ISM? early-AGB wind? instrumental scattered light?) remains unclear.

This preliminary, qualitative description enhances the complexity of NGC 6741 (a common characteristic of all PNe analysed at adequate spatial and spectral resolutions). Before starting a thorough investigation of the kinematical and physical information contained in the spectra, we underline that the general characteristics of NGC 6741 - in particular: (a) nebular compactness and (b) star weakness (the stellar continuum, generally used as a position marker, is absent in the echellograms) - make this nebula the ideal target to test the reliability limits of our 3-D reconstruction procedure.

\section{The gas spatio-kinematics}

According to Sabbadin et al. (2004, and references therein), the overall spatio-kinematical properties of a regularly expanding nebula can be derived by combining the kinematics of the gas projected at the apparent position of the star (the "central star pixel line", cspl, of the echellograms, common at all PA) with the spatial profile at the systemic radial velocity (the "zero velocity pixel column", zvpc, at each PA).

Although the stellar continuum is absent in the echellograms of NGC 6741, we obtain a satisfactory cspl-location and zvpc-alignement (within $\pm 0.1^{\prime \prime}$ ) of the echelle orders thanks to: (a) the central, symmetrical position of the star in the broad-band HST-WFPC2 nebular images; (b) the cspl- and zvpc-calibration given by the continuum spectrum of the central star of NGC 7009 (observed in the same night, and with the same instrumental configuration).

The peak separations in the cspl of NGC $6741,2 V_{\text {exp }}$, are contained in Table 1 (last column), where ions are put in 
Table 1. Peak separation in the cspl of NGC 6741.

\begin{tabular}{ccccc}
\hline \hline Ion & IP range $(\mathrm{eV})$ & \multicolumn{4}{c}{$2 V_{\exp }\left(\mathrm{km} \mathrm{s}^{-1}\right)$} \\
\cline { 3 - 5 } & & Wilson $(1950)$ & Robinson et al. (1982) & This paper \\
\hline$[\mathrm{O} \mathrm{I}]$ & $0.0-13.6$ & - & - & 55.1 \\
{$[\mathrm{~S} \mathrm{II}]$} & $10.4-23.3$ & - & - & 54.0 \\
{$[\mathrm{O}$ II $]$} & $13.6-35.1$ & 44.2 & - & 53.7 \\
H I & $\geq 13.6$ & & - & 44.0 \\
{$[\mathrm{~N} \mathrm{II}]$} & $14.5-29.6$ & 42.1 & - & 53.5 \\
{$[\mathrm{~S} \mathrm{III}]$} & $23.4-34.8$ & - & - & 48.2 \\
$\mathrm{He}$ I & $24.6-54.4$ & - & - & 48.0 \\
{$[\mathrm{Ar}$ III $]$} & $27.6-40.7$ & - & - & 47.6 \\
{$[\mathrm{O} \mathrm{III}]$} & $35.1-54.9$ & 41.6 & 42 & 46.1 \\
{$[\mathrm{Ar} \mathrm{IV}]$} & $40.7-59.8$ & - & - & 33.7 \\
{$[\mathrm{Ne} \mathrm{III]}$} & $41.0-63.4$ & 41.0 & - & 48.2 \\
$\mathrm{~N} \mathrm{III}$ & $47.4-77.5$ & - & - & 40.0 \\
$\mathrm{He} \mathrm{II}$ & $\geq 54.4$ & - & - & 34.0 \\
{$[\mathrm{Ar} \mathrm{V}]$} & $59.8-75.0$ & - & - & 25.0 \\
{$[\mathrm{Ne} \mathrm{V}]$} & $97.1-126.2$ & $0.0:$ & - & - \\
\hline
\end{tabular}

order of increasing IP. Typical errors are $1.0 \mathrm{~km} \mathrm{~s}^{-1}$ for the strongest forbidden emissions (like $\lambda 4959-5007 \AA$ of [O III] and $\lambda 6548-6584 \AA$ of [N II]) to $2.0 \mathrm{~km} \mathrm{~s}^{-1}$ for the faintest ones (in particular, $\lambda 6312 \AA$ of [S III], $\lambda 4711-4740 \AA$ of [Ar IV] and $\lambda 6435-7005 \AA$ of [Ar V]). The uncertainties for recombination lines are: $2.0 \mathrm{~km} \mathrm{~s}^{-1}$ for $\lambda 4861-6563 \AA$ of $\mathrm{H} \mathrm{I}$, $\lambda 4686-6560 \AA$ of He II and $\lambda 4640 \AA$ of N III, and $1.5 \mathrm{~km} \mathrm{~s}^{-1}$ for $\lambda 5876-6678 \AA$ of He I.

The agreement with the kinematical results given in the literature (also contained in Table 1) is quite poor, probably due to the compactness of NGC 6741, combined with image rotation on the slit (long-exposure Coudé spectra by Wilson 1950) or inadequate spatial resolution (circular aperture $18^{\prime \prime}$ in diameter by Robinson et al. 1982).

Concerning the zvpc at the nine PA of NGC 6741, the intensity peak separations, $2 r_{\mathrm{zvpc}}$, in the different ionic species are listed in Table 2.

To assemble the kinematical results of Table 1 and the spatial results of Table 2 we can follow two ways:

(I) Search for the PA with $\boldsymbol{R}_{\mathrm{zvpc}} \simeq \boldsymbol{R}_{\mathrm{cspl}}$. Let us assume for the main shell of NGC 6741 the most general spatial structure, i.e. a tri-axial ellipsoid with axes $a, b$ and $c$. At PA = $75^{\circ}$ to $115^{\circ}$ (close to the apparent major axis, Fig. 4) the line-tilt indicates that the observer is not aligned with the major axis, and the overall emission structure (suggestive of a dense equatorial torus + two faint polar lobes) excludes the oblate $(a=b)$ ellipsoid hypothesis. Moreover, the absence of line-tilt at $\mathrm{PA}=15^{\circ}$ (perpendicular to the apparent major axis, Fig. 3) means that either the minor axis of the ellipsoid is close to PA $=15^{\circ}$, or the intermediate axis of the ellipsoid is close to PA $=15^{\circ}$, or the ellipsoid is prolate $(b=c)$. In all cases we conclude that, to a first approximation, $R$ (minor axis) $\simeq R$ (intermediate axis $) \simeq R_{\text {zvpc }}\left(\mathrm{PA}=15^{\circ}\right) \simeq R_{\mathrm{cspl}}$, providing (through Tables 1 and 2): $V_{\exp }\left(\mathrm{km} \mathrm{s}^{-1}\right)=13( \pm 2) \times R^{\prime \prime}$.
(II) Spectral image deformation along the major axis. Let us consider a generic, expanding $\left(V_{\exp } \propto R\right)$ tri-axial ellipsoid denser in the equatorial region than at the poles, seen at an intermediate direction. A spectrograph slit aligned with the apparent major axis intercepts the radial slice of nebula shown in Fig. 7 (left panel): an ellipse with polar axis $a$ and equatorial axis $b$, perpendicular to each other. The corresponding spectral image is an ellipse too (Fig. 7, central panel), but deformed (with respect to the tomographic map) by the telescope + spectrograph characteristics. Note that:

- the polar axis $\left(a^{\prime}\right)$ and the equatorial axis $\left(b^{\prime}\right)$ of the spectral image are no longer perpendicular to each other;

- the original tomographic map (Fig. 7, left panel) can be obtained by means of a simple compression of the spectral image (Fig. 7, central panel) along the $x$ (i.e. velocity) axis, untill $a^{\prime} \perp b^{\prime 1}$.

In practice, and reversing the foregoing procedure, we compress the [O III] spectral image of NGC 6741 at $\mathrm{PA}=75^{\circ}$ (Fig. 7 , central panel) by a factor 1.60 along the $x$ axis, thus correcting for the spectral deformation introduced by NTT+EMMI on the nebular slice intercepted by the slit, and obtaining:

- the tomographic map shown in Fig. 7 (left panel);

- the expansion law $V_{\text {exp }}\left(\mathrm{km} \mathrm{s}^{-1}\right)=13( \pm 1) \times R^{\prime \prime}$.

This is the same law given by method (I). The compression factor along the $x$ axis being the same at all PA, we can repeat the procedure for the [O III] spectral image of NGC 6741 at $\mathrm{PA}=15^{\circ}$ (close to the apparent minor axis; Fig. 3), thus recovering the tomographic map shown in the right panel of Fig. 7, i.e. an almost circular ring with $R_{\mathrm{zvpc}}\left(\mathrm{PA}=15^{\circ}\right) \simeq R_{\mathrm{cspl}}, \mathrm{QED}^{2}$.

1 This visual effect can be seen by rotating Fig. 7 around the $y$ axis.

2 quod erat demostrandum; Latin for: which was to be proved. 
Table 2. Peak separation in the zvpc at the nine PA of NGC 6741.

\begin{tabular}{|c|c|c|c|c|c|c|c|c|c|}
\hline \multirow[t]{2}{*}{ Ion } & \multicolumn{9}{|c|}{ (arcsec) } \\
\hline & $\mathrm{PA}=15^{\circ}$ & $35^{\circ}$ & $55^{\circ}$ & $75^{\circ}$ & $95^{\circ}$ & $115^{\circ}$ & $135^{\circ}$ & $155^{\circ}$ & $175^{\circ}$ \\
\hline [O I] & 4.3 & 4.6 & 5.7 & 7.3 & 6.8 & 7.1 & 6.1 & 5.6 & 4.6 \\
\hline [S II] & 4.1 & 4.5 & 5.5 & 7.1 & 6.6 & 6.9 & 6.0 & 5.5 & 4.4 \\
\hline [O II] & 4.0 & 4.3 & 5.2 & 6.6 & 6.5 & 6.6 & 5.8 & 5.2 & 4.3 \\
\hline HI & 3.5 & 3.5 & 4.1 & 6.0 & 6.0 & 6.0 & 5.2 & 4.5 & 3.8 \\
\hline [N II] & 4.1 & 4.4 & 5.4 & 6.8 & 6.5 & 6.5 & 5.9 & 5.2 & 4.4 \\
\hline [S III] & 3.6 & 4.1 & 4.6 & 6.0 & 5.7 & 6.0 & 5.5 & 4.6 & 3.9 \\
\hline $\mathrm{He} \mathrm{I}$ & 3.8 & 4.0 & 4.5 & 6.2 & 5.7 & 6.0 & 5.5 & 4.6 & 4.0 \\
\hline [Ar III] & 3.7 & 3.8 & 4.5 & 5.9 & 5.0 & 6.0 & 5.5 & 4.6 & 4.1 \\
\hline [O III] & 3.6 & 3.7 & 4.4 & 5.3 & 4.7 & 5.5 & 5.0 & 4.4 & 3.8 \\
\hline [Ar IV] & 2.7 & 3.2 & 3.6 & 3.9 & 3.9: & 5.3: & 4.5: & 3.4 & 3.1 \\
\hline [Ne III] & 3.6 & 3.5: & 4.0 & 4.5 & $5.0:$ & 5.6: & 5.0 & 4.0 & 3.6 \\
\hline N III & 3.0: & 3.1: & 3.6: & 4.0: & 4.2: & 5.1: & 4.4: & $3.4:$ & 3.2: \\
\hline He II & 2.6 & 2.9 & 3.4 & 3.8 & 3.9: & 5.0: & 3.9 & 3.2 & 2.9 \\
\hline [Ar V] & 2.3 & 2.6 & 2.8 & 3.0 & 3.5: & 4.0: & 3.6 & 3.0 & 2.7 \\
\hline
\end{tabular}
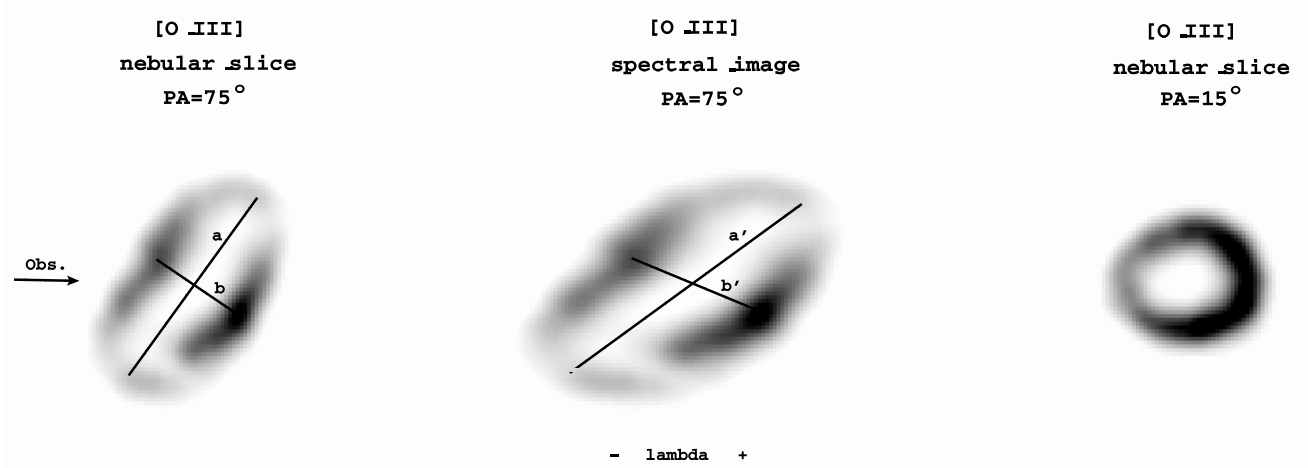

Fig. 7. Nebular tomography vs. spectral image connection. In this example we use the [O III $]$ spectral images of NGC 6741 at PA $=75^{\circ}$ and $\mathrm{PA}=15^{\circ}$ (same orientation as Figs. 3 and 4). Left panel: tomographic map of the nebular slice intercepted by a spectrograph slit aligned with the apparent major axis of an ellipsoidal PN denser at the equator than at the poles; $a$ and $b$ are the polar and equatorial axes, respectively, of the elliptical nebular slice. Central panel: spectral image of the nebular slice shown in the left panel; $a^{\prime}$ and $b^{\prime}$ are the polar and equatorial axes, respectively, of the spectral image. Right panel: [O III] tomographic reconstruction of NGC 6741 at PA $=15^{\circ}$. See the text for details.

The detailed cspl-zvpc relation for NGC 6741 at $\mathrm{PA}=15^{\circ}$, shown in Fig. 8, indicates that:

a) on the whole, the ionized gas follows Wilson's law: the high-excitation zones expand more slowly than the lowexcitation ones, and a positive correlation exists between the expansion velocity and the size of the monochromatic image (Wilson 1950);

b) the range of both $2 r_{\mathrm{zvpc}}$ and $2 V_{\exp }$ is quite large (2.3 to $4.3 \operatorname{arcsec}$ and 25.0 to $55.1 \mathrm{~km} \mathrm{~s}^{-1}$, respectively), suggesting a broad radial density profile and large stratification of the radiation;

c) the general expansion law, $V_{\exp }\left(\mathrm{km} \mathrm{s}^{-1}\right)=13 \times R^{\prime \prime}$, fails in the innermost, highest ionization layers marked by the [Ar V] emissions: they expand slower than expected.

Point c) is quite peculiar among PNe, and deserves some comment. Deceleration is present (and clear) in both the $\lambda 6435 \AA$ and $\lambda 7005 \AA$ [Ar V] lines at all PA of NGC 6741 (except along and close to the apparent major axis, where the open-ended structure of the $[\mathrm{ArV}]$ spectral image prevents any conclusion; see Fig. 4, lower-right panel), whereas no evidence of deceleration appears in other high ionization species, like He II (due to the blurred emission) and N III ( $\lambda 4640 \AA$ As too faint). Although a detailed spatio-kinematical study at even higher ionization stages appears indispensable - for example in the forbidden line of $\mathrm{Ne}^{+4}(\mathrm{IP}=97.1 \mathrm{eV}$ ) at $\lambda 3425 \AA$ (which is outside our spectral range) - support for the deceleration hypothesis comes from the classical work by Wilson (1950), who obtained $V_{\text {exp }}[\mathrm{Ne} \mathrm{V}] \simeq 0 \mathrm{~km} \mathrm{~s}^{-1}$ (quite uncertain).

We believe that NGC 6741 is the first PN showing clear evidence of deceleration in the highest ionization layers (the second candidate being IC 418: a preliminary analysis of our NTT+EMMI echellograms, taken at six PA, suggests the presence of infalling gas).

Vice versa, recent results by Gesicki \& Zijlstra (2003, $3 \mathrm{PNe}$ ) and Gesicki et al. (2003, $14 \mathrm{PNe})$ - based on highresolution emission profiles integrated along the slit, and spherical shell hypothesis for the emitting gas - indicate that acceleration is a quite common property in the PN innermost 


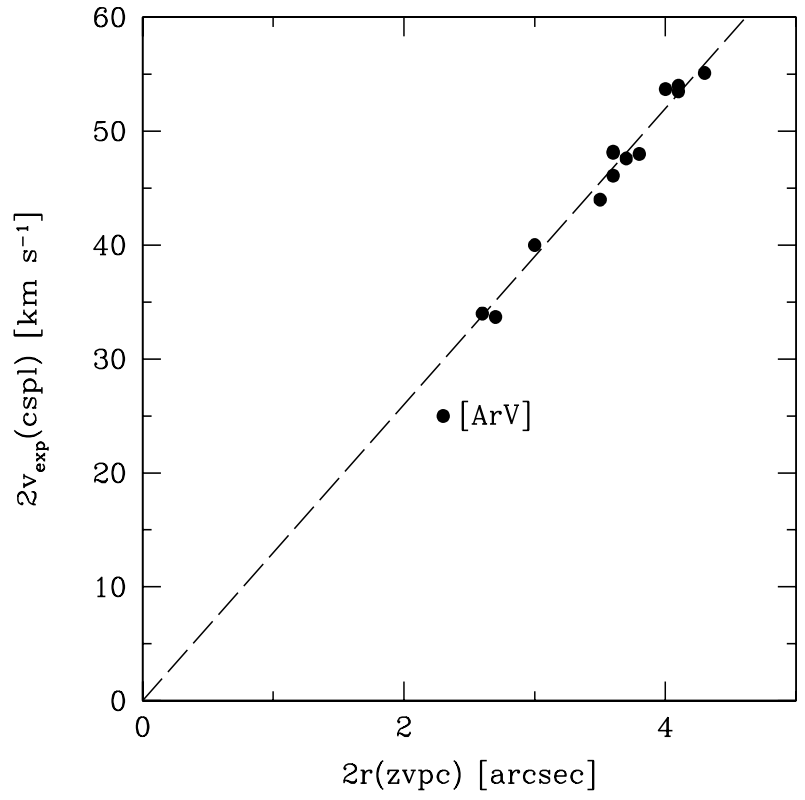

Fig. 8. The cspl-zvpc relation for NGC 6741 at PA $=15^{\circ}$, superimposed on the adopted expansion law $V_{\exp }\left(\mathrm{km} \mathrm{s}^{-1}\right)=13 \times R^{\prime \prime}$, which is valid across the whole nebula, except in the innermost, highestionization, decelerated regions marked by [Ar V].

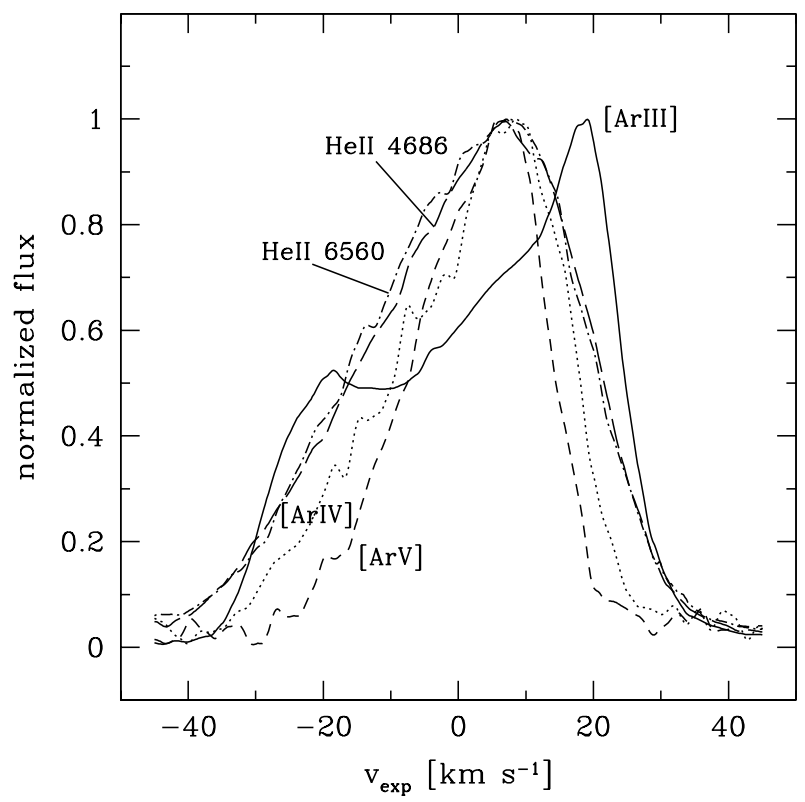

Fig. 9. Selected emission line profiles integrated along the slit for NGC 6741 at $\mathrm{PA}=15^{\circ}$, showing the spurious kinematical results provided by the recombination lines of He II. Symbols: continuous line $=$ [Ar III] at $\lambda 7135 \AA$; dotted line $=$ [Ar IV] at $\lambda 4740 \AA$; short-dashed line $=[\mathrm{Ar} \mathrm{V}]$ at $\lambda 7005 \AA$; long-dashed line $=\mathrm{He}$ II at $\lambda 4686 \AA$; dotteddashed line $=\mathrm{He}$ II at $\lambda 6560 \AA$. For details, see the text.

layers (i.e. "U"-shaped expansion profile), due to the dynamical contribution by the shocked, hot wind from the central star. Gesicki \& Zijlstra (2003) adopted $\lambda 4686 \AA$ of He II as diagnostic of the high-ionization kinematics, whereas Gesicki et al. (2003) used $\lambda 5007 \AA$ of [O III] (in a few cases $\lambda 6560 \AA$ of He II).
The following caveats are in order:

1) the spherical shell assumption is not accurate;

2) $\lambda 5007 \AA$ of [O III] (IP range 35.1 to $54.9 \mathrm{eV}$ ) is a poor diagnostic of the highest ionization strata (except for very-low excitation $\mathrm{PNe}$ );

3) the recombination lines of hydrogen and helium suffer severe blurring effects (thermal motion, fine-structure and expansion velocity gradient across the nebula combined with the small number of ionization stages of $\mathrm{H}$ and $\mathrm{He}$ ), introducing spurious kinematical results.

In particular, $\lambda 4686 \AA$, He II Paschen $\alpha$, consists of thirteen fine-structure components spread in the $\lambda \lambda 4685.377-4685.918 \AA$ range (corresponding to $\Delta V=34.6 \mathrm{~km} \mathrm{~s}^{-1}$ ), with a strong blue-shifted tail (see Figs. 3 and 4). In the case of $\lambda 6560 \AA$, He II Pickering $\beta$, there are nineteen fine-structure components covering the $\lambda \lambda 6559.769-6560.209 \AA$ spectral range $\left(\Delta V=20.1 \mathrm{~km} \mathrm{~s}^{-1}\right)$.

To further test this point, let us consider the emission line profiles integrated along the slit of NGC 6741 at $\mathrm{PA}=15^{\circ}$. The results for $\lambda 4686 \AA$ and $\lambda 6560 \AA$ of He II, and the ionic sequence of argon - [Ar III] at $\lambda 7135 \AA,[\mathrm{Ar}$ IV] at $\lambda 4740 \AA$ and [Ar V] at $\lambda 7005 \AA$ - are shown in Fig. 9; they confirm that:

- a) spherical symmetry is a simplistic assumption;

- b) the He II recombination lines fail to fit the welldefined stratification of both the radiation and expansion present in the ionic sequence of argon, $F W H M(\mathrm{He}$ II, IP $>54.4 \mathrm{eV})$ being intermediate between $F W H M([\mathrm{Ar} \mathrm{III}]$, IP range 27.6-40.7 eV) and FWHM([ArIV], IP range 40.7-59.8 eV), whereas, according to the detailed kinematical results presented in this section, we would expect $F W H M(\mathrm{He}$ II $) \leq F W H M([\mathrm{Ar}$ IV $]) \ll F W H M([\mathrm{Ar}$ III $])$.

The same discrepancy occurs when considering either more parameters (like full-width at $10 \%$ maximum flux), different ionic sequences, further PA of NGC 6741, or other PNe of our sample.

A direct confirmation of the misleading kinematical results - in particular, the high-velocity of the innermost layers - provided by the combination (spectral profile of recombination lines integrated along the slit) + (spherical symmetry assumption) comes from a comparative analysis of the emission line profiles contained in Gesicki \& Zijlstra (2003) and Gesicki et al. (2003): in all cases (17 PNe) the forbidden lines of the ionic species with the highest IP also show the sharpest emission profile (as expected for a simple, positive $V_{\exp }$ vs. radius relation).

All this questions the general validity of the spatiokinematical studies based on the spherical symmetry assumption and/or spectral profiles of recombination lines integrated along the slit, and weakens (or even cancels) the reliability of their results on turbulence, radial kinematics, matter distribution and ionization, whose quantification needs detailed studies at high spatial and spectral resolutions.

Figure 10 (multi-color in the electronic version) shows the complete velocity field at the nine observed PA of NGC 6741. We select He II, [O III] and [NII] as markers of the high, medium and low-excitation regions, respectively. These 


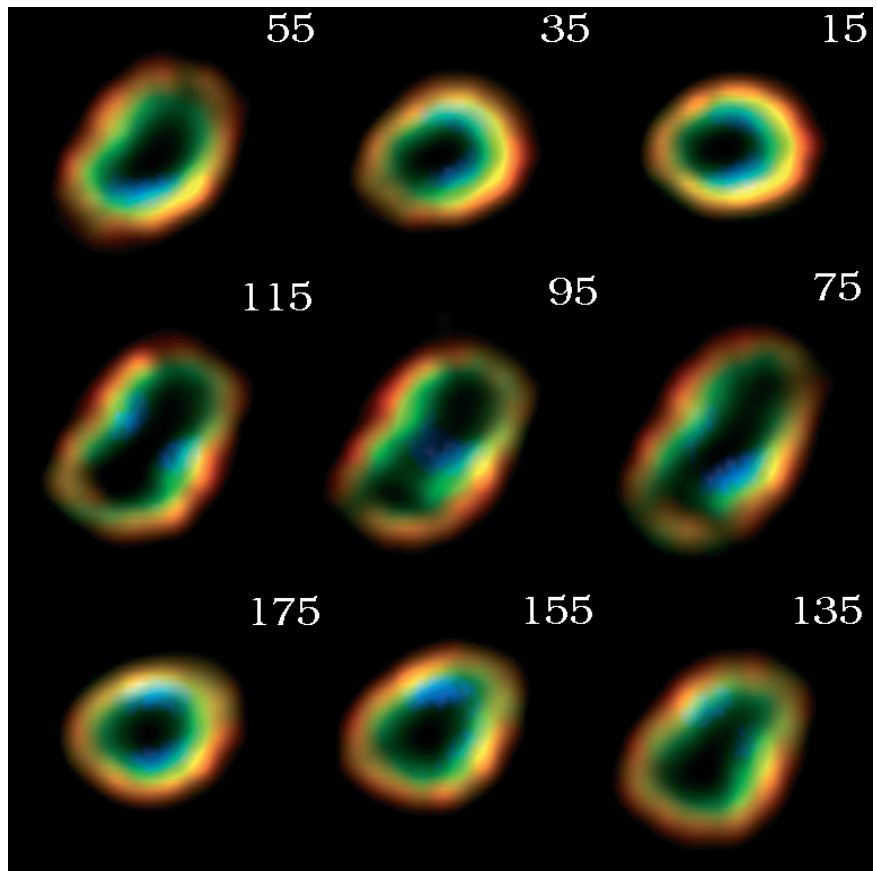

Fig. 10. Combined position-velocity maps in the nine observed PA of NGC 6741 at high (He II), medium ([O III]) and low ([N II]) excitation (multi-color maps in the electronic version of the paper; blue = He II, green $=[\mathrm{OIII}]$, and red $=[\mathrm{NII}])$, scaled according to the relation $V_{\exp }\left(\mathrm{km} \mathrm{s}^{-1}\right)=13 \times R^{\prime \prime}$. The orientation of these tomographic maps is the same of Figs. 3 and 4.

position-velocity $(P-V)$ maps are relative to the systemic heliocentric velocity of the nebula, $V_{\text {rad } \odot}=$ $+39.6( \pm 1.0) \mathrm{km} \mathrm{s}^{-1}$, corresponding to $V_{\mathrm{LSR}}=$ $+56.4( \pm 1.0) \mathrm{km} \mathrm{s}^{-1}$, and are scaled (along the $x$ axis) according to $V_{\exp }\left(\mathrm{km} \mathrm{s}^{-1}\right) \simeq 13 \times R^{\prime \prime}$, i.e. they reproduce the tomographic maps of the nebular slices covered by the spectrograph slit.

Figure 10 highlights the kinematical complexity and large stratification of the radiation within NGC 6741. The main nebula consists of an almost-prolate ellipsoid ( $a \simeq 7.4^{\prime \prime}, a / b \simeq 1.8$, $a / c \simeq 2.0$ ), whose major axis (projected at $\mathrm{PA} \simeq 95^{\circ}$ ) forms an angle of $55( \pm 3)^{\circ}$ with the line of sight: on the whole, the eastern part of the nebula is approaching the observer, and the western part receding.

All this throws new light on two specific fields: (I) nature of the curious skip of absorbing knots present in Fig. 1 (upper panel), and (II) turbulent motion.

(I) Skip of absorbing knots: the central location, orientation (vertical) and light curvature (concavity towards East) are suggestive of an inhomogeneous belt of neutral matter (gas + molecules + dust) embedding the dense ionized gas of the equatorial regions. The amount of circum-nebular neutral gas can be estimated from the [O III] flux-depletion suffered by the underlying ionized nebula, being:

$\log \frac{F(5007)_{\text {off-knot }}}{\mathrm{F}(5007)_{\text {on-knot }}}=k_{5007}-k_{\mathrm{H}_{\beta}}+c(\mathrm{H} \beta)_{\text {circum-nebular }}$, where $k_{\lambda}$ is the extinction coefficient (Seaton 1979), and $c(\mathrm{H} \beta)_{\text {circum-nebular }}$ the logarithmic extinction at $\mathrm{H} \beta$ caused by the local absorbing matter.

Intensity scans in the neighbourhood of the deepest knots provide $\frac{F(5007)_{\text {off-knot }}}{F(5007)_{\text {on-knot }}}$ up to $1.4( \pm 0.1)$, i.e. $c(\mathrm{H} \beta)_{\text {circum-nebular }}$ up to $0.18( \pm 0.03)$. Assuming a "normal" gas-to-dust ratio (Spitzer 1978; Bohlin et al. 1978) and $c(\mathrm{H} \beta)=1.48 \times E(B-V)($ Acker 1978), we have:

$n(\mathrm{HI})=\frac{4.8 \times 10^{21} \times E(B-V)_{\text {circum-nebular }}}{\Delta l}$,

where $n(\mathrm{HI})$ is the $\mathrm{HI}$ density (atoms $\mathrm{cm}^{-3}$ ), and $\Delta l$ the radial thickness $(\mathrm{cm})$ of the absorbing layer. For $D=2000 \mathrm{pc}$ (see Sect. 5) and $\Delta l=[r($ halo $)-r$ (main nebula at PA $=$ $\left.\left.15^{\circ}\right)\right] / 2 \simeq 2.0^{\prime \prime}$ (from Figs. 1 and 2 ), we obtain an indicative value of $n(\mathrm{HI}) \simeq 7 \times 10^{3}$ atoms cm $\mathrm{cm}^{-3}$. Such a high density of the circum-nebular matter in NGC 6741 is further support for the recombination hypothesis outlined in the previous sections.

(II) Turbulent motion. In Fig. 10, the tomographic map close to the apparent minor axis (i.e. at PA $=15^{\circ}$ ) being almost circular and quite homogeneous, we can assume $W(\text { spat })_{\text {intrinsic }}\left(\mathrm{zvpc}\right.$ at $\left.\mathrm{PA}=15^{\circ}\right) \simeq W(\mathrm{vel})_{\text {intrinsic }}(\mathrm{cspl})$, thus inferring $W(\text { vel })_{\text {turb }}$ (through Eqs. (1) to (3), $V_{\exp }\left(\mathrm{km} \mathrm{s}^{-1}\right)=$ $13 \times R^{\prime \prime}$, and the $T_{\mathrm{e}}$ radial profile given in Sect. 7.1). We overlook the recombination lines of hydrogen and helium (dominated by thermal motion and/or fine-structure), and consider the strongest forbidden lines. The analysis of $\lambda 6300 \AA$ ([O I]), $\lambda 6731 \AA$ ([S II]), $\lambda 6584 \AA$ ([N II]), $\lambda 7135 \AA$ ([Ar III]), and $\lambda 5007 \AA$ ([O III] $)$ provides $W(\mathrm{vel})_{\text {turb }}=3.5( \pm 2.0) \mathrm{km} \mathrm{s}^{-1}$, with no evident relation to the ionization degree. Thus, in spite of the crude assumptions and wide uncertainties, we conclude that the turbulent motion in NGC 6741 is quite modest.

\section{The absorption (interstellar + circum-nebular)}

In general, the observed line intensities must be corrected for absorption according to:

$\log \frac{I(\lambda)_{\mathrm{corr}}}{I(\lambda)_{\mathrm{obs}}}=k_{\lambda} \times c(\mathrm{H} \beta)_{\mathrm{tot}}$

where $k_{\lambda}$ is the extinction coefficient (Seaton 1979), and $c(\mathrm{H} \beta)_{\text {tot }}$ the logarithmic extinction at $\mathrm{H} \beta$ given by:

$c(\mathrm{H} \beta)_{\mathrm{tot}}=c(\mathrm{H} \beta)_{\text {interstellar }}+c(\mathrm{H} \beta)_{\text {circum-nebular }}$

with $c(\mathrm{H} \beta)_{\text {circum-nebular }}=0$ for an optically thin, fully ionized, density bounded nebula. Decidedly, this is not the case of NGC 6741, which is optically thick, ionization bounded, and wrapped up in a dense cocoon of almost-neutral matter.

The extinction estimates reported in the literature (mean values along the nebular slice covered by the spectrograph slit) cluster around $c(\mathrm{H} \beta)_{\text {tot }}=1.05$ (Kaler \& Lutz 1985; Kaler \& Jacoby 1989; Cahn et al. 1992; Hyung \& Aller 1997).

To disentangle the complex absorption over NGC 6741, we apply the $\mathrm{H} \alpha / \mathrm{H} \beta$ analysis of the whole spectral image, as introduced by Turatto et al. (2002). Figure 11 shows the $F(\mathrm{H} \alpha) / F(\mathrm{H} \beta)$ isophotal contours superimposed on the $\mathrm{H} \beta$ spectral image for three representative PA of NGC 6741: at 

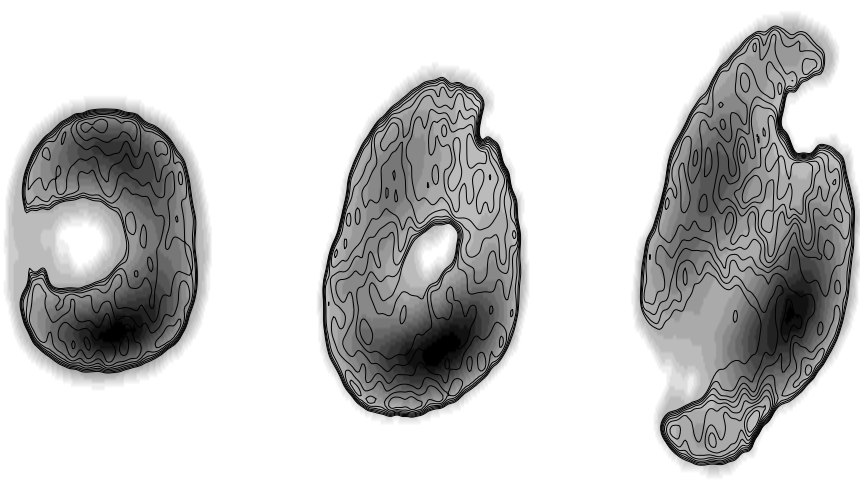

Fig. 11. $F(\mathrm{H} \alpha) / F(\mathrm{H} \beta)$ isophotal contours superimposed to the $\mathrm{H} \beta$ spectral image for three representative PA of NGC 6741. Left panel: $\mathrm{PA}=15^{\circ}$ (close to the apparent minor axis); central panel: $\mathrm{PA}=55^{\circ}$ (intermediate direction); right panel: $\mathrm{PA}=75^{\circ}$ (close to the apparent major axis). Same orientation as Figs. 3 and 4. The isophotal contours cover the range 5.20 (the outermost) to 7.60, with a constant step of 0.30 .

$\mathrm{PA}=15^{\circ}$ (close to the apparent minor axis; untilted $\mathrm{H} \beta$ spectral image) $F(\mathrm{H} \alpha) / F(\mathrm{H} \beta)$ increases outwards along the spatial (i.e. $y$ ) axis, peaking beyond the top of the $\mathrm{H} \beta$ flux. At $\mathrm{PA}=55^{\circ}$ (intermediate $\mathrm{PA}$; tilted $\mathrm{H} \beta$ spectral image) the $F(\mathrm{H} \alpha) / F(\mathrm{H} \beta)$ rise along the $y$ axis is overwhelmed by the broad maximum to the S-W of the nebular centre (at the expected position of the absorbing belt visible in Fig. 1, upper panel). The same occurs at $\mathrm{PA}=75^{\circ}$ (close to the apparent major axis; tilted $\mathrm{H} \beta$ spectral image), where $F(\mathrm{H} \alpha) / F(\mathrm{H} \beta)$ peaks W-SW of the centre, corresponding to the equatorial absorbing belt.

Summing up the $\mathrm{H} \alpha / \mathrm{H} \beta$ intensity distribution at the nine observed PA of NGC 6741, we infer that:

a) $c(\mathrm{H} \beta)_{\text {interstellar }}=0.95( \pm 0.05)$,

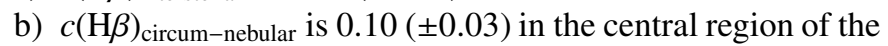
nebular image (out of the equatorial absorbing belt), and rises to $0.20( \pm 0.05)$ within the equatorial absorbing belt, and at the edge of the ionized zone.

Moreover, a modest decrease of $F(\mathrm{H} \alpha) / F(\mathrm{H} \beta)$ in the innermost regions (out of the equatorial absorbing belt) is suggestive of a local increase of $T_{\mathrm{e}}$.

Last, an indicative value of the total neutral mass embedding the ionized nebula (through Eq. (5) and using simple geometrical considerations $)$ is $M_{\text {neutral }} \simeq 0.20( \pm 0.05) M_{\odot}$.

Although the small angular size prevents a deeper analysis, these results do represent the umpteenth sign of the complex structure and peculiar evolutionary phase of NGC 6741, and will be a precious support in the determination of the topical parameter, i.e. distance.

\section{The nebular distance, size and age}

The "statistical" distance of NGC 6741, provided by two dozen catalogues using different methods and assumptions, is:

$\langle D\rangle($ Shklovsky $) \simeq 3300( \pm 1000) \mathrm{pc}$

$\langle D\rangle($ ionized mass-radius relation $) \simeq 1500( \pm 700) \mathrm{pc}$

$\langle D\rangle($ other methods $) \simeq 2000( \pm 1000) \mathrm{pc}$, where $\langle D\rangle$ (Shklovsky) represents an upper limit of the true distance, NGC 6741 being an optically thick, ionization bounded PN.

Individual values reported in the literature are: $1300 \mathrm{pc}$ (nebular radial velocity combined with the circular law of galactic rotation; Acker 1978), 2100 pc (Acker 1978) and $1400 \mathrm{pc}$ (Pottasch 1983) (both based on the largescale galactic interstellar absorption map by Lucke 1978), and $1500 \mathrm{pc}$ (color-excess vs. distance relation for early-type stars within $1.5^{\circ}$ of the nebula; Kaler \& Lutz 1985).

We tried to determine the nebular parallax by combining the expansion velocity field with the angular expansion measured in first- and second-epoch HST-WFPC2 frames. The NASA public archives contain 21 images of NGC 6741, taken at two epochs separated by 2.97 years (programs GO 7501 and GO 8773; P. I. Arsen Hajian). The [O III] and [N II] multiple exposures (WFPC2 central planetary camera, PC; pixel size $=0.0455$ arcsec) were co-added, corrected for optical distortions, aligned and rotated using IRAF packages (Reed et al. 1999; Palen et al. 2002).

No apparent image-shift is detected, i.e. image-shift $\leq$ $\frac{1}{2}$ pixel, and $\frac{\mathrm{d} \theta}{\mathrm{d} t} \leq 8 \times 10^{-3} \operatorname{arcsec}_{\mathrm{yr}}{ }^{-1}$. For an optically thin, fully ionized PN this implies $D \geq 1400$ pc, being:

$D(\mathrm{pc})=\frac{0.211 V_{\mathrm{exp}}\left(\mathrm{km} \mathrm{s}^{-1}\right)}{\frac{\mathrm{d} \theta}{\mathrm{d} t}\left(\operatorname{arcsec} \mathrm{yr}^{-1}\right)}$

But NGC 6741 is optically thick (probably recombining), and Eq. (8) cannot be applied, since in this case we should compare the expansion velocity of the ionized gas with the angular expansion of the ionization front. In the limiting case of a nearby nebula in a very deep recombination phase, we even expect a detectable contraction of the ionization front. Thus, we infer that $D(\mathrm{NGC} 6741) \geq 1500 \mathrm{pc}$, and/or the nebula is at the end of the recombination phase (or at the reionization start).

We decided to enrich (and sharpen, if possible) the broad color-excess vs. $D$ relation given by Kaler \& Lutz (1985) with recent bibliographic reports. Scanning the NGC 6741 neighbourhood with the SIMBAD facilities of CDS (Centre de Données astronomiques de Strasbourg) gave two dozen (lowweight) field stars with accurate photometry and spectral type (in most cases the luminosity class is absent; we assume a luminosity class V), and two important distance markers:

- the open cluster OCL 88 (at an apparent distance of 15.4' from NGC 6741), characterized by $E(B-V)=1.0$ and $D=$ 3000 pc (Sagar \& Griffiths 1998, and referenced therein),

- the classical Cepheid V336 Aql (apparent distance from NGC $\left.6741=37.0^{\prime}\right)$, with $\log P=0.8636$ days, $\left\langle m_{V}\right\rangle=$ 9.875, $E(B-V)=0.61-0.64$, and $D=1800-2100 \mathrm{pc}$ (Pel 1976; Feast \& Whitelock 1997; Metzger et al. 1998; Berdnikov et al. 2000).

The improved $E(B-V)$ vs. $D$ relation in the direction of NGC 6741 is shown in Fig. 12: the interstellar absorption starts at $D \simeq 200-300 \mathrm{pc}$, and gradually increases up to $3000 \mathrm{pc}$ (and beyond). This is in partial disagreement with literature reports. NGC 6741 is within the Aquila-Scutum cloud, a galactic region characterized by a high, quite uniform star density, suggestive 


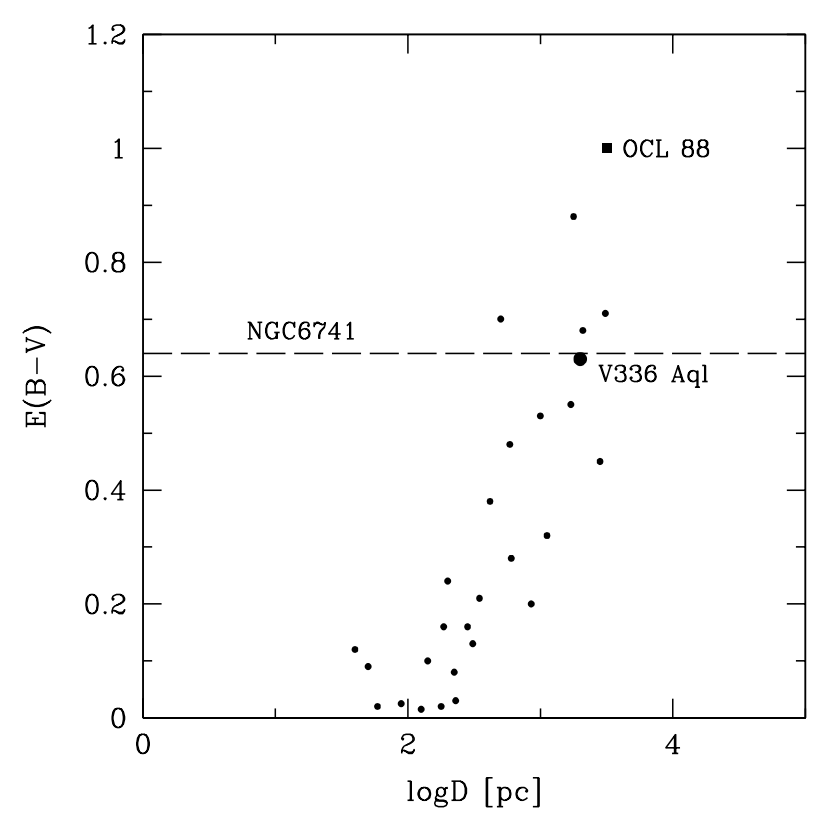

Fig. 12. Color-excess vs. distance relation in the direction of NGC 6741. Symbols: small dots $=$ field stars; filled square $=$ open cluster OCL 88; large dot = classical Cepheid V336 Aql.

of a low obscuration. According to Forbes (1985), it shows an almost immediate rise at $A_{V}=2.3 \mathrm{mag}$. within $300 \mathrm{pc}$; beyond this distance, there is little or no increase of extinction out to $D \simeq 6000 \mathrm{pc}$.

To test this point, we consider the four more PNe projected within the Aquila-Scutum cloud (at an apparent distance from NGC 6741 less than 90'). They are CBSS 3, K 3-19, M 1-66 and K 3-20 (we reject a fifth object, Sp 2-151, because of the unreliable line intensities - in particular $F(\mathrm{H} \alpha) / F(\mathrm{H} \beta) \simeq 1.0$ - and peculiar spectral-type A for the exciting star reported by Acker et al. 1992, probably due to the partial blending with a relatively bright, $m_{R} \simeq 13.0$, field star). The four selected $\mathrm{PNe}$ in the Aquila-Scutum cloud have $1.5 \leq c(\mathrm{H} \beta) \leq 2.2$ (line intensities by Acker et al. 1992; and Cappellaro et al. 1994, for $T_{\mathrm{e}}=10^{4} \mathrm{~K}$ and $N_{\mathrm{e}}=2 \times 10^{3} \mathrm{~cm}^{-3}$ ), i.e. $3.2 \leq A_{V} \leq 4.7$, and, using the obscuration law by Forbes (1985), $D>6000 \mathrm{pc}$ for all four nebulae.

The last result appears quite improbable. Thus, in the following we will adopt the color-excess vs. distance relation shown in Fig. 12 and $D($ NGC 6741) $=2000( \pm 300)$ pc.

The linear size of the main nebula is $0.036 \mathrm{pc} \times 0.020 \mathrm{pc} \times$ $0.018 \mathrm{pc}$ (major, intermediate and minor semi-axes, respectively), whereas both the spherical recombining halo and the [O III] rays punching the [N II] skin close to the apparent major axis extend up to $0.080 \mathrm{pc}$.

The "kinematical" age of NGC 6741, $t_{\text {kin }}=R / V_{\exp }$, is about 750 years and the "true" age $t_{\text {true }} \simeq 2 R /\left[V_{\text {exp }}\right.$ (today) + $\left.V_{\text {exp }}(\mathrm{AGB})\right] \simeq 1400$ years.

Summing up: NGC 6741 is a very young, compact, high surface brightness (i.e. dense) PN. The combination of (a) high excitation (up to [Ne V]) of the innermost layers; (b) a low ionization skin; and (c) an almost neutral, large density halo, is indicative of a very hot central star at low luminosity. A deep analysis of the powering engine is needed.

\section{The central star parameters}

The HST-WFPC2 frames of NGC 6741 taken through the broad-band filter $F 555 W$ (central wavelength $=5407 \AA$, bandwidth $=1236 \AA$ ) provide $m_{V}=20.09( \pm 0.05)$, where the unknown star color is the main source of inaccuracy. Previous ground-based estimates reported in the literature are: 19.5 (Pottasch 1981), 17.6 (Tylenda et al. 1989), 19.16 (Kaler \& Jacoby 1989) and 19.26 (Hyung \& Aller 1997) for $m_{V}$, and $>20.2$ (Gathier \& Pottasch 1988) and 18.2 (Tylenda et al. 1989) for $m_{B}$.

The $\mathrm{H}$ I and He II Zanstra temperatures are given by the stellar magnitude, combined with both the total $\mathrm{H} \beta$ nebular flux, $\log F(\mathrm{H} \beta)_{\mathrm{obs}}=-11.32( \pm 0.03) \mathrm{mW} \times \mathrm{m}^{-2}$ (Kaler \& Lutz 1985; Kaler \& Jacoby 1989; Acker et al. 1991; Cahn et al. 1992; this paper), and the flux ratio $F(\lambda 4686 \AA) / F(\mathrm{H} \beta)=$ $0.40( \pm 0.03)$ (Aller et al. 1985; Kaler \& Jacoby 1989; Hyung $\&$ Aller 1997; this paper).

We obtain $\log \left(T_{\mathrm{Z}} \mathrm{HI}\right)=5.33( \pm 0.07)$ and $\log \left(T_{\mathrm{Z}} \mathrm{He}\right.$ II $)=$ $5.23( \pm 0.07)$, thus confirming the peculiarity already reported by Heap et al. (1989) and Hyung \& Aller (1997), i.e. the Zanstra discrepancy is reversed. $T_{\mathrm{Z}} \mathrm{HI}>T_{\mathrm{Z}} \mathrm{He}$ II is a typical signature of recombining PNe, e.g. NGC 6565 (Turatto et al. 2002), where the ionization and thermal structure are out of equilibrium, and the recombination processes dominate. These are faster for the higher-ionization species (Tylenda 1986; Stasinska 1989; Marten \& Szczerba 1997); thus, in the following, we will adopt $T_{*} \simeq T_{\mathrm{Z}} \mathrm{He}$ II $<T_{\mathrm{Z}} \mathrm{H} \mathrm{I}$.

The stellar luminosity (using $D=2000 \mathrm{pc}$, and the bolometric corrections by Schönberner 1981) is $\log L_{*} / L_{\odot}=$ $2.75( \pm 0.15)$.

The high temperature and low luminosity of the star, added to the low nebular age, suggest that the stellar mass, $M_{*}$, is larger than the average value $\left(\simeq 0.60 M_{\odot}\right)$ of the PNe nuclei. According to the evolutionary tracks by Schönberner (1981, 1983), Iben (1984), Wood \& Faulkner (1986), Blöcker \& Schönberner (1990), Vassiliadis \& Wood (1994) and Blöcker (1995), the $0.66-0.68 M_{\odot}$ post-AGB star of NGC 6741 has recently (a few hundreds years ago) exhausted the hydrogen-shell nuclear burning, and is fading along the white dwarf cooling sequence.

The early luminosity decline of the star was very fast (and caused the nebular recombination), but later it gradually slowed, so that we cannot exclude that, at present, the gas has reached (or even passed) the contraction-expansion equilibrium condition, $(1 / 3) \times\left(\mathrm{d}\left(\ln L_{*}\right) / \mathrm{d} t\right)=-2 / t_{\text {kin }}$ in the classical Strömgren model, thanks to matter dilution due to expansion.

New input will come from the nebular physical conditions, radial ionization structure and photo-ionization model.

\section{The nebular parameters}

\subsection{Physical conditions and ionized mass}

Following Sabbadin et al. (2004, and references therein), the radial profile of the physical conditions is given by the zvpc, which is independent of the expansion velocity field, since it represents the gas in the plane of the sky, whose motion is tangential. For $T_{\mathrm{e}}$ we use the classical diagnostic line ratios of 
ions in $\mathrm{p}^{2}$ and $\mathrm{p}^{4}$ configurations $(\lambda 5007 \AA / \lambda 4363 \AA$ of [O III] and $\lambda 6584 \AA / \lambda 5755 \AA$ of [N II]; Aller 1984; Osterbrock 1989), whereas $N_{\mathrm{e}}$ comes from both diagnostic line ratios of ions in the $\mathrm{p}^{3}$ configuration $(\lambda 6717 \AA / \lambda 6731 \AA$ of [S II] and $\lambda 4711 \AA / \lambda 4740 \AA$ of [Ar IV]), and the absolute $\mathrm{H} \alpha$ flux distribution.

The main limitation is connected to the NGC 6741 compactness, large stratification of the radiation and weakness of the [N II] auroral line and the [S II] and [ArIV] doublets: $T_{\mathrm{e}}[\mathrm{N} \mathrm{II}], N_{\mathrm{e}}[\mathrm{S} \mathrm{II}]$ and $N_{\mathrm{e}}[\mathrm{Ar}$ IV] can be derived only at (or close to) the intensity peak of the corresponding emission.

Moreover, the large $\mathrm{H} \alpha$ broadening implies a complex deconvolution for instrumental resolution plus thermal motion plus fine-structure (see Sect. 2), lowering the accuracy of the $F(\mathrm{H} \alpha)_{\text {zvpc }}$ and $N_{\mathrm{e}}(\mathrm{H} \alpha)$ profiles. Thus, according to Benetti et al. (2003), $F(\mathrm{H} \alpha)_{\text {zvpc }}$ and $N_{\mathrm{e}}(\mathrm{H} \alpha)$ are also obtained from the radial ionization structure relative to $\mathrm{O}^{++}$(Sect. 7.2) and the fair assumption $\mathrm{O} / \mathrm{H}=$ const. across the nebula; at each position:

$$
\frac{F(\mathrm{H} \alpha)_{\mathrm{zvpc}}}{F(\lambda 5007 \AA)_{\mathrm{zvpc}}} \propto \frac{\mathrm{H}}{\mathrm{O}} \times f\left(T_{\mathrm{e}}\right) \times \operatorname{icf}\left(\mathrm{O}^{++}\right),
$$

with icf $\left(\mathrm{O}^{++}\right)=\frac{\mathrm{O}}{\mathrm{O}^{++}}=$ionization correcting factor.

For the internal, high-excitation regions $\operatorname{icf}\left(\mathrm{O}^{++}\right)$comes from the ionization structure of helium (Seaton 1968; Benetti et al. 2003):

$\operatorname{icf}\left(\mathrm{O}^{++}\right)_{\text {inner }}=1+\frac{0.3 \times \mathrm{He}^{++}}{\mathrm{He}^{+}} ;$

for the external, low-excitation layers we adopt:

$\operatorname{icf}\left(\mathrm{O}^{++}\right)_{\text {outer }}=1+\frac{\mathrm{O}^{0}}{\mathrm{O}^{++}}+\frac{\mathrm{O}^{+}}{\mathrm{O}^{++}}$,

which includes the ionization effects produced by the resonant charge-exchange reaction $\mathrm{O}^{+}+\mathrm{H}^{0} \leftrightarrows \mathrm{O}^{0}+\mathrm{H}^{+}$; according to Osterbrock (1989) and Stancil et al. (1999), in the outermost nebular regions $\mathrm{H}^{0} / \mathrm{H}^{+} \simeq 0.83 \times\left(\mathrm{O}^{0} / \mathrm{O}^{+}\right)$.

Moreover (Sabbadin et al. 2004),

$N_{\mathrm{e}}(\mathrm{H} \alpha) \propto \frac{1}{T_{\mathrm{e}}^{-0.47}} \times\left(\frac{F(\mathrm{H} \alpha)_{\mathrm{zvpc}}}{\epsilon_{\mathrm{l}} \times D}\right)^{1 / 2}$,

where $D$ is the (known) distance, and $\epsilon_{1}$ the (un-known) "local filling factor", i.e. the fraction of the local volume actually filled by matter with density $N_{\mathrm{e}}$.

A comparative analysis gives quite satisfactory results: the $N_{\mathrm{e}}(\mathrm{H} \alpha)$ profiles obtained in the two ways differ by less than $5 \%$ everywhere, except in the faint, innermost regions, where the discrepancy rises up to $10 \%$. In the following we will adopt $N_{\mathrm{e}}(\mathrm{H} \alpha)$ given by $F(\lambda 5007 \AA)_{\text {zvpc }}$ and Eqs. (9) to (12).

Figure 13 shows the resulting radial distribution of the physical conditions in NGC 6741 at $\mathrm{PA}=15^{\circ}$ and $\mathrm{PA}=75^{\circ}$ (close to the apparent minor and major axes, respectively).

The radial trend of $T_{\mathrm{e}}[\mathrm{OIII}]$ is common at all PA: $\geq 15000 \mathrm{~K}$ in the weak innermost regions, rapidly decreasing outward to $12000 \mathrm{~K}$ in the densest layers, and more or less constant further (uncertain). $T_{\mathrm{e}}[\mathrm{N} \mathrm{II}]$ is systematically lower than $T_{\mathrm{e}}[\mathrm{O} \mathrm{III}]$. All this is in quantitative agreement with the results by Hyung \& Aller (1997) and Pottasch et al. (2001).

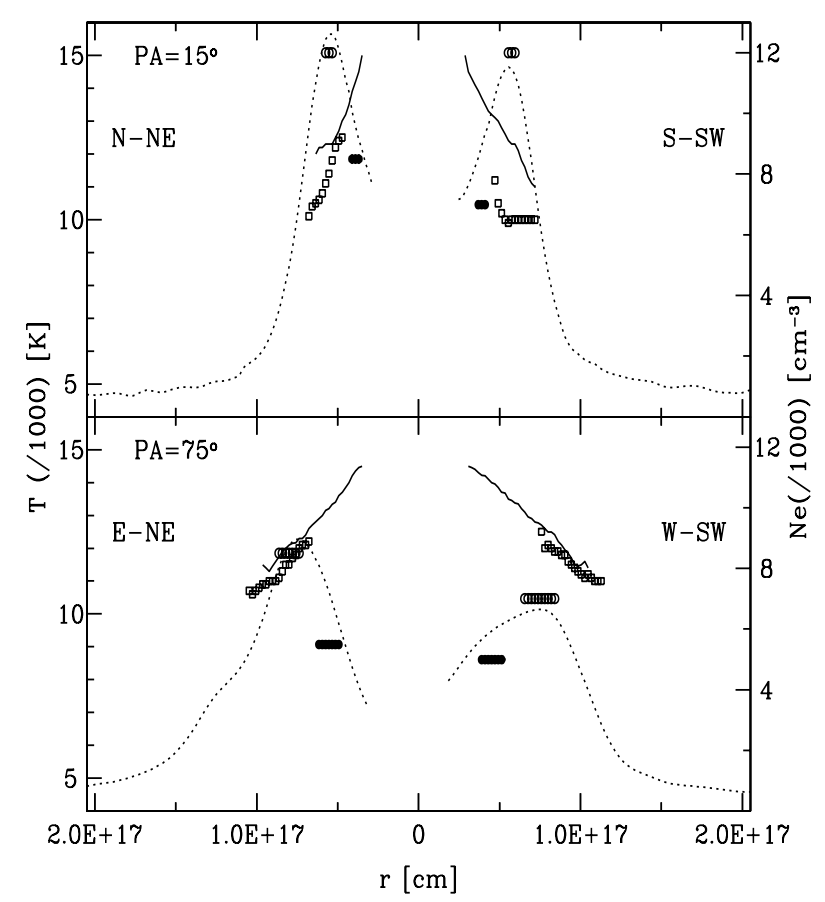

Fig. 13. Radial distribution of the physical conditions $\left(T_{\mathrm{e}}\right.$ and $\left.N_{\mathrm{e}}\right)$ at two selected directions of NGC 6741. Upper panel: the zvpc at PA = $15^{\circ}$ (close to the apparent minor axis); lower panel: the zvpc at $\mathrm{PA}=$ $75^{\circ}$ (close to the apparent major axis). Left ordinate scale: $T_{\mathrm{e}}[\mathrm{O}$ III] (continuous line) and $T_{\mathrm{e}}[\mathrm{N} \mathrm{II}]$ (empty squares). Right ordinate scale: $N_{\mathrm{e}}(\mathrm{H} \alpha)$ for $D=2000 \mathrm{pc}$ (dotted line), $N_{\mathrm{e}}[\mathrm{S}$ II] (empty circles), and $N_{\mathrm{e}}[\mathrm{Ar}$ IV] (filled circles).

$N_{\mathrm{e}}$ presents a broad, asymmetric bell-shape profile with peaks up to $12000( \pm 1000) \mathrm{cm}^{-3}$ close to the minor axis, and $6000-8000( \pm 800) \mathrm{cm}^{-3}$ close to the major axis. Note that, in spite of the high density peaks - causing strong collisional de-excitation of the [S II] $\lambda 6717 \AA$ line - we have $N_{\mathrm{e}}[\mathrm{S} \mathrm{II}] \simeq N_{\mathrm{e}}(\mathrm{H} \alpha)$, i.e. the local filling factor is $\epsilon_{\mathrm{l}} \simeq 1$, with $N_{\mathrm{e}}[\mathrm{S} \mathrm{II}] \times \epsilon_{1}^{0.5} \simeq N_{\mathrm{e}}(\mathrm{H} \alpha)$ (Aller 1984; Pottasch 1984; Osterbrock 1989).

$N_{\mathrm{e}}\left[\mathrm{Ar}\right.$ IV] is systematically lower than $N_{\mathrm{e}}[\mathrm{S}$ II] adopting the electron impact excitation rates by Keenan et al. (1997), whereas $N_{\mathrm{e}}[\mathrm{Ar} \mathrm{IV}] \simeq N_{\mathrm{e}}[\mathrm{S} \mathrm{II}]$ for earlier collisional rates (for example, Aller 1984).

Concerning the halo, $N_{\mathrm{e}}$ peaks at the inner edge $\left(\simeq 1000-1500 \mathrm{~cm}^{-3}\right)$, and decreases outwards.

Recent density determinations (mean values integrated over the slit) reported in the literature for NGC 6741 are: $N_{\mathrm{e}}$ (different ions) $=6300 \mathrm{~cm}^{-3}$ by Hyung \& Aller (1997), $N_{\mathrm{e}}[\mathrm{S} \mathrm{II}]=6000 \mathrm{~cm}^{-3}, N_{\mathrm{e}}[\mathrm{OII}]=8500 \mathrm{~cm}^{-3}, N_{\mathrm{e}}[\mathrm{Cl} \mathrm{IIII}]=$ $9000 \mathrm{~cm}^{-3}$ and $N_{\mathrm{e}}\left[\mathrm{Ar}\right.$ IV] $=5500 \mathrm{~cm}^{-3}$ by Pottasch et al. (2001), and $N_{\mathrm{e}}[\mathrm{Cl} \mathrm{III}]=4470 \mathrm{~cm}^{-3}, N_{\mathrm{e}}[\mathrm{Ar} \mathrm{IV}]=6610 \mathrm{~cm}^{-3}$ and $N_{\mathrm{e}}[52 \mu \mathrm{m} / 88 \mu \mathrm{m}]=2880 \mathrm{~cm}^{-3}$ by Liu et al. (2001).

The ionized mass of NGC 6741, obtainable from the observed $N_{\mathrm{e}}$ spatial distribution, the $\mathrm{H} \beta$ flux, and the radio flux (Aller 1984; Pottasch 1984; Osterbrock 1989; and Turatto et al. $2002)$, results to be $M_{\text {ion }} \simeq 0.06( \pm 0.02) M_{\odot}$, i.e. much lower than the mass of the external, neutral cocoon, $M_{\text {neutral }} \simeq 0.20$ $( \pm 0.05) M_{\odot}$ (see Sect. 4), thus confirming the peculiar evolutionary phase of our nebula. 
The relatively high $N_{\mathrm{e}}$ of the halo implies a quite recent recombination start, no more that 200 years ago. In fact, the $N_{\mathrm{e}}$ depletion rate for recombination is:

$\mathrm{d} N_{\mathrm{e}} / \mathrm{dt}=-\alpha_{\mathrm{B}} \times N_{\mathrm{e}} \times N\left(\mathrm{H}^{+}\right)$,

with $\alpha_{\mathrm{B}}=$ effective recombination coefficient. Assuming $N_{\mathrm{e}} \simeq$ $N\left(\mathrm{H}^{+}\right)$and $T_{\mathrm{e}}=12000 \mathrm{~K}$, and neglecting the recombination delay due to expansion, we obtain:

$N_{\mathrm{e}}(0)=N_{\mathrm{e}}(t) /\left[1-8.2 \times 10^{-6} \times t \times N_{\mathrm{e}}(t)\right]$,

where $N_{\mathrm{e}}(0)$ is the initial electron density, and $N_{\mathrm{e}}(t)$ the electron density at time $t$ (in years) elapsed from the recombination start. Adopting $N_{\mathrm{e}}(0) \simeq 10000 \mathrm{~cm}^{-3}$ (mean of the density peaks in Fig. 13), and $N_{\mathrm{e}}(t) \simeq 600 \mathrm{~cm}^{-3}$ (the lowest value in Fig. 13), we have $t \simeq 190$ years. A very similar "recombination age" is inferred from the mean H I density of the circumnebular matter (Sect. 3).

All this, combined with the short nebular age ( $\simeq 1400$ years), agrees with the theoretical evolutionary times of a $0.66-0.68 M_{\odot}$ post-AGB star. According to Blöcker (1995, and references therein), in the interval $t_{\mathrm{SW}}$ (end of the superwind ejection) to $t_{\mathrm{SW}}+1100 \mathrm{yr}$ the hydrogen-burning star horizontaly crosses the H-R diagram (at $\log L_{*} / L_{\odot} \simeq 4.00$ ) becoming hotter and hotter (up to $\log T_{*} \simeq 5.20 \mathrm{~K}$ ). At $t_{\mathrm{SW}}+1100 \mathrm{yr}$ the nuclear fuelling becomes insufficient, and in a century the luminosity declines to $\log L_{*} / L_{\odot} \simeq 3.70$ (whereas $T_{*}$ reaches its maximum, $\log T_{*} \simeq 5.30 \mathrm{~K}$ ). At $t_{\mathrm{SW}}+1200 \mathrm{yr}$ the hydrogen-shell burning ceases, the stellar evolution drastically accelerates untill $t_{\mathrm{SW}}+1400 \mathrm{yr}$ $\left(\log L_{*} / L_{\odot} \simeq 2.80 ; \log T_{*} \simeq 5.20 \mathrm{~K}\right)$, and later slows down (for example, at $t_{\mathrm{SW}}+3000 \mathrm{yr}$ we have $\log L_{*} / L_{\odot} \simeq 2.40$ and $\log T_{*} \simeq 5.15 \mathrm{~K}$ ).

Thus, NGC 6741 is close to (or has even passed) the recombination-reionization transition.

\subsection{Radial ionic and mean chemical abundances and photo-ionization model}

The zvpc of the different emissions furnishes the detailed radial ionization structure of NGC 6741. Since the blurred $\mathrm{H} \alpha$ appearance lowers the accuracy of the $F(\mathrm{H} \alpha)_{\mathrm{zvpc}}$ distribution, we adopt $\lambda 5007 \AA$ as the reference emission, thus inferring the radial ionization structure relative to $\mathrm{O}^{++}$(for details, see Benetti et al. 2003). The $\frac{\mathrm{X}^{+i}}{\mathrm{O}^{++}}$profiles of NGC 6741 at $\mathrm{PA}=15^{\circ}$ and $\mathrm{PA}=75^{\circ}$ (close to the apparent minor and major axes, respectively), presented in Fig. 14, confirm that the nebula is optically thick in all directions.

Within this scenario, the [O III] rays punching the [N II] skin (Fig. 2) identify a few radial directions along and close to the true major axis of the nebula with "low" $N_{\mathrm{e}}$ peaks $\left(N_{\mathrm{e}}\right.$ (peak) $\leq 4000 \mathrm{~cm}^{-3}$ according to the photo-ionization model presented in this section): in these directions (barely visible in our spectral images at $\mathrm{PA}=75^{\circ}, 95^{\circ}$ and $115^{\circ}$; see Figs. 4 and 10) recombination processes are less efficient and the gas can be (partially) ionized by the UV stellar flux at larger distances.

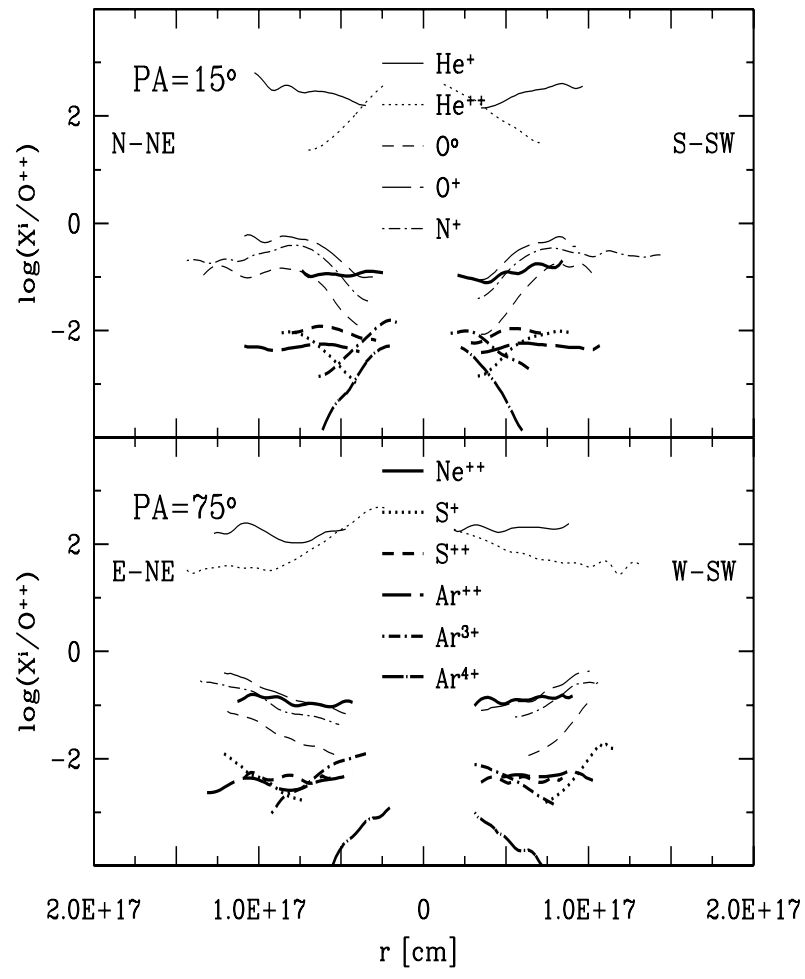

Fig. 14. The $\frac{\mathrm{X}^{+i}}{\mathrm{O}^{++}}$radial ionization structure of NGC 6741 in the zvpc at $\mathrm{PA}=15^{\circ}$ (close to the apparent minor axis; upper panel), and $\mathrm{PA}=$ $75^{\circ}$ (close to the apparent major axis; lower panel). Same orientation as Fig. 13.

When combined with the results in the previous sections, the overall, complex structure of NGC 6741, fully driven by the fast evolving central star, can be divided into three distinct zones showing quite different physical characteristics: the internal, high-to-medium excitation nebula directly ionized by the stellar radiation ends in a sharp transition zone at mediumto-low excitation marking the present edge of the ionization front. It is surrounded by the gas no longer reached by the fading UV flux (i.e. the halo), dominated by recombination processes. A similar onion-like radial structure (ionizationtransition-recombination) is observed in NGC 6565 (Turatto et al. 2002).

Although the ionization and thermal structure of NGC 6741 are out of equilibrium, the large $N_{\mathrm{e}}$ of the ionized gas (peaks up to $12000 \mathrm{~cm}^{-3}$ ) assures a rapid nebular response to the variable stellar flux. In particular, the delay in the "transition zone" is short, the recombination time, $t_{\mathrm{rec}}=1 /\left(\alpha_{\mathrm{B}} \times N_{\mathrm{e}}\right)$, being a few years for hydrogen (even shorter for higher ionization species).

Thus, NGC 6741 is nearly in equilibrium. This allows us to neglect (to a first approximation) the time dependance, and estimate the total chemical abundances of the ionized gas with the classical method valid for "static" nebulae.

We follow a two-step procedure:

I (broad approach): according to the critical analyses by Alexander \& Balick (1997) and Perinotto et al. (1998), the mean ionic abundances are obtained from the line fluxes integrated over the spatial profile and the expansion velocity field; later on, we correct for the unobserved ionic stages by means of the ionization correcting factors, derived both empirically 
Table 3. NGC 6741: chemical abundances (relative to $\log H=12$ ).

\begin{tabular}{lcccccccc}
\hline \hline Reference & $\mathrm{He}$ & $\mathrm{C}$ & $\mathrm{N}$ & $\mathrm{O}$ & $\mathrm{Ne}$ & $\mathrm{S}$ & $\mathrm{Cl}$ & $\mathrm{Ar}$ \\
\hline Hyung \& Aller (1997) (icf) & 11.01 & 8.86 & 8.38 & 8.73 & 8.12 & 6.89 & 5.43 & 6.49 \\
Hyung \& Aller (1997) (model B) & 11.04 & 8.90 & 8.15 & 8.65 & 8.00 & 6.76 & 5.34 & 6.54 \\
Pottasch et al. (2001) & 11.04 & 8.81 & 8.45 & 8.82 & 8.26 & 7.04 & - & 6.69 \\
Pottasch et al. (2002) & 11.04 & 8.56 & 8.26 & 8.65 & 8.18 & 6.90 & 5.26 & 6.51 \\
Perinotto et al. (2004b) & 11.08 & - & 8.31 & 8.70 & 8.16 & 6.70 & - & 6.54 \\
This paper (icf) & 11.04 & - & 8.20 & 8.66 & 8.08 & 6.78 & - & 6.54 \\
This paper (CLOUDY) & 11.04 & - & 8.28 & 8.66 & 8.08 & 6.90 & - & 6.54 \\
\hline
\end{tabular}

Table 4. Input parameters for the model nebula (CLOUDY).

\begin{tabular}{|c|c|}
\hline Radial density profile & $\begin{array}{l}\text { ionized gas = Fig. } 13 ; \text { cf. Sect. } 7.1 \\
\text { neutral gas }=\text { cf. Sects. } 3 \text { and } 4\end{array}$ \\
\hline $\begin{array}{l}\text { Chemical abundances: } \\
\mathrm{C}, \mathrm{Cl}, \mathrm{K}, \mathrm{Ca}, \mathrm{Mg}, \mathrm{Si} \\
\mathrm{He}, \mathrm{N}, \mathrm{O}, \mathrm{Ne}, \mathrm{S}, \mathrm{Ar} \\
\text { other elements }\end{array}$ & $\begin{array}{l}\text { Hyung \& Aller (1997) model B } \\
\text { Table } 3 \text { (second row) } \\
\text { PN (CLOUDY default) }\end{array}$ \\
\hline Dust & PN (CLOUDY default) \\
\hline Local filling factor & 1.0 \\
\hline Exciting star & $\begin{array}{l}\text { blackbody distribution } \\
T_{*}=170000 \mathrm{~K} \\
\log L_{*} / L_{\odot}=2.75\end{array}$ \\
\hline Distance & $2.0 \mathrm{kpc}$ \\
\hline
\end{tabular}

(Barker 1983, 1986) and from interpolation of theoretical nebular models (Shields et al. 1981; Aller \& Czyzak 1983; Aller 1984; Osterbrock 1989). The resulting mean chemical abundances are listed in Table 3 (second row); recent estimates reported in the literature are also given in the table.

II (refining): we apply the photo-ionization code CLOUDY (Ferland et al. 1998) to a model nebula characterized by the same distance, gas distribution, mean chemical composition and exciting star parameters as NGC 6741 and combine the model-line profiles (convolved for a seeing+guiding of $0.60^{\prime \prime}$ ) with the line profiles observed in the true nebula.

Let us focus on the zvpc of the S-SW sector at PA $=15^{\circ}$ (close to the apparent minor axis of NGC 6741). The input parameters of the model-nebula are given in Table 4, and Fig. 15 shows the physical conditions in the model nebula (to be compared with Fig. 13), and the absolute radial flux distribution of the main emission in both the model nebula and NGC 6741.

Figure 15 provides a satisfactory model nebula vs. true nebula agreement for the internal, high-to-medium excitation regions and the transition zone. Further, the weak recombining halo appears in NGC 6741, whereas emission is absent in the static model nebula. The same is observed at the other PA.
In more detail, some disturbing drifts arise in Fig. 15. Two minor discrepacies are:

(a) uncertain flux distribution of [Ne III] in NGC 6741 (ascribable to the edge location of $\lambda 3968 \AA$ in the original NTT+EMMI frames);

(b) comparable model nebula vs. true nebula flux-shift for [S II] and [S III], indicating a slight underestimate of sulphur abundance in Table 3 (second row), and in Table 4 (model nebula). The same occurs for [N II]. The improved chemical abundances of NGC 6741 are reported in the last row of Table 3 .

The two major problems in Fig. 15 concern:

(1) the ionization structure of helium: He I $\lambda 5876 \AA$ and He II $\lambda 4686 \AA$ are weaker and stronger, respectively, in the model nebula than in the true nebula;

(2) the peak emission and whole flux distribution of the highest excitation ions (He II, [ArIV] and [Ar V]) tend to be systematically closer to the central star in the true nebula than in the model nebula.

Ad hoc manipulation of the model nebula input data lowers or even cancels - the foregoing problems; for example, we can act on the ionizing UV distribution, given the strong $T_{*}$ dependance of both the ionic structure of helium and the radial profile of the highest-excitation emissions. In fact, the only decrease by $17 \%$ of the stellar temperature (i.e. for $T_{*} \simeq 150000 \mathrm{~K}$, which is within the observational error box given in Sect. 6) simultaneously relieves discrepancies (1) and (2). They almost disappear with a further, modest increase (by 20\%) of the matter density in the innermost nebular layers.

Unfortunately, the large number of parameters involved (density profile, chemical abundances, dust, temperature and luminosity of the star) and assumptions (distance, black-body distribution, seeing convolution), added to the nebular compactness and peculiar evolutionary phase, greatly complicate the analysis, whose definitive solution needs detailed observations at even higher spatial and spectral resolutions.

\section{The 3-D morpho-kinematical structure}

According to the reconstruction method introduced by Sabbadin et al. $(1985,1987)$ and deepened by Sabbadin et al. (2000a,b), we select $\lambda 4686 \AA$ of He II, $\lambda 5007 \AA$ of [O III] 


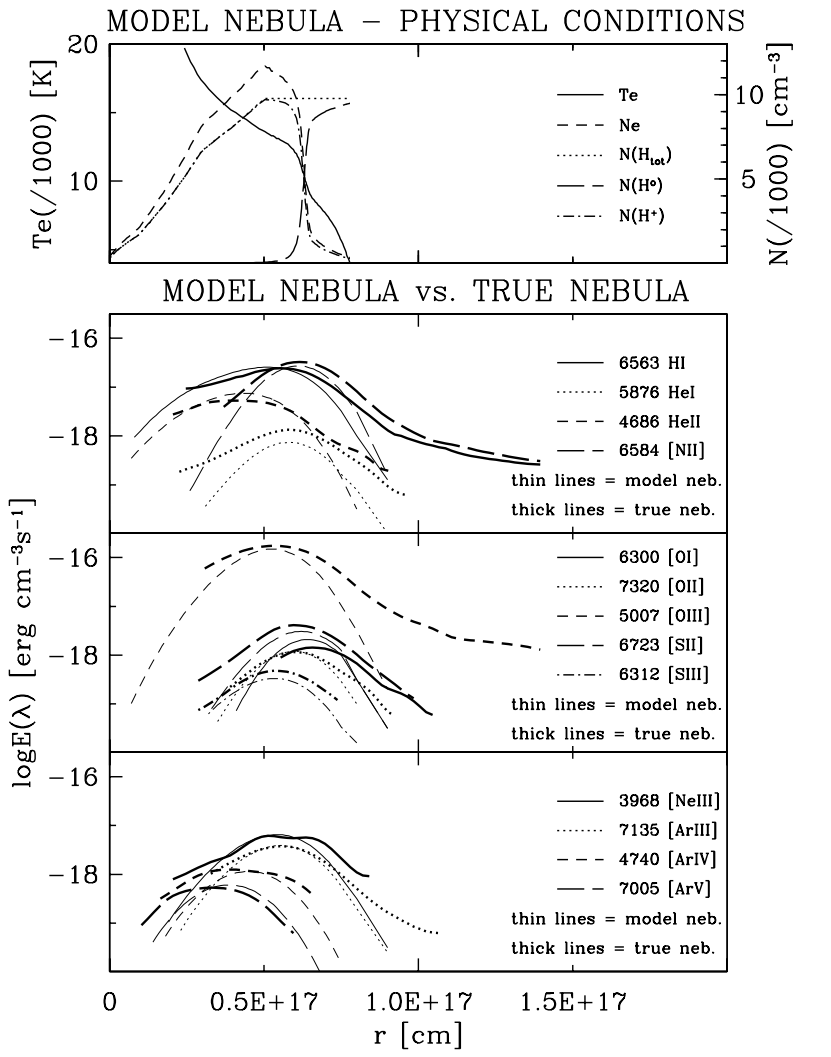

Fig. 15. Model-nebula (CLOUDY) vs. true-nebula (NGC 6741, $\mathrm{PA}=15^{\circ}, \mathrm{S}-\mathrm{SW}$ sector$)$ : physical conditions and radial ionization structure. Top panel: physical conditions in the model-nebula (to be compared with Fig. 13). Second panel: absolute flux distribution of HI $\lambda 6563 \AA$, [N II] $\lambda 6584 \AA$, and the ionic sequence of helium (He I $\lambda 5876 \AA$ and He II $\lambda 4686 \AA$ ) in the model nebula (thin symbols) and the true nebula (thick symbols). Third panel: absolute flux distribution for the ionic sequences of oxygen ([O I] $\lambda 6300 \AA$, [O II] $\lambda 7320 \AA$, and [O III] $\lambda 5007 \AA)$ and sulphur ([S II] $\lambda 6717+6731 \AA$, and [S III] $\lambda 6312 \AA$ ); same symbols as in the second panel. Bottom panel: absolute flux distribution for [Ne III] $\lambda 3968 \AA$, and the ionic sequence of argon ([Ar III] $\lambda 7135 \AA$, [Ar IV] $\lambda 4740 \AA$, and [Ar V] $\lambda 7005 \AA$ ); same symbols as in the second and third panels.

and $\lambda 6584 \AA$ of $[\mathrm{N} \mathrm{II}]$ as markers of the high, medium and low-ionization regions of NGC 6741, respectively. The spectral images are:

(a) de-convolved for seeing, spectral resolution and thermal motion (fine-structure also is taken into account for the recombination line of $\mathrm{He}$ II);

(b) de-projected through the relation $V_{\exp }\left(\mathrm{km} \mathrm{s}^{-1}\right)=13 \times R^{\prime \prime}$ (see Sect. 3);

(c) assembled by means of the 3-D rendering procedure described by Ragazzoni et al. (2001).

Here we present a limited number of frames, corresponding to a partial rotation around the North-South axis centered on the exciting star (i.e. almost perpendicular to the major axis). The complete series of nebular movies is available:

- in the electronic version of the paper (on-line data);
- at http://web.pd.astro.it/sabbadin, the WEB site dedicated to the 3-D structure of expanding nebulae;

- at http://www.edpsciences.org.

Figures 16 and 17 show the opaque reconstruction of NGC 6741 in He II, [O III] and [N II] at high and low fluxlevels, respectively, for a rotation of $180^{\circ}$ through the first Euler angle. The $(0,0)$ images correspond to the Earth-nebula direction (North is up and East to the left). An indication of the $N_{\mathrm{e}}$ limit in Figs. 16 and 17 can be obtained from the corresponding [O III] absolute flux-cut: $\log E(\lambda 5007 \AA)=-15.95$ and $-16.70 \mathrm{erg} \mathrm{s}^{-1} \mathrm{~cm}^{-3}$ (high and low cuts, respectively). Since $\mathrm{O} / \mathrm{H}=4.6 \times 10^{-4}($ Table 3$), \operatorname{icf}\left(\mathrm{O}^{++}\right) \simeq 1.1$ and $N_{\mathrm{e}} \simeq$ $1.15 \times N\left(\mathrm{H}^{+}\right)$, they correspond to $N_{\mathrm{e}}$ (high cut) $\simeq 9000 \mathrm{~cm}^{-3}$ for Fig. 16 and $N_{\mathrm{e}}$ (low cut) $\simeq 4000 \mathrm{~cm}^{-3}$ for Fig. 17 (assuming $T_{\mathrm{e}}=12000 \mathrm{~K}$ and $\epsilon_{\mathrm{l}}=1$ ).

The projection of NGC 6741 for a rotation around the $\mathrm{N}-\mathrm{S}$ direction (almost perpendicular to the major axis) is presented in Fig. 18 (multi-color images in the electronic version), providing a representative sample of the nebular appearance when changing the line of sight. The left panel, $(0,0)$, corresponds to NGC 6741 as seen from Earth (North is up and East to the left), to be compared with Fig. 1 and the HST image by Hajian \& Terzian at http://ad.usno.navy.mil/pne/gallery.html

The overall characteristics of NGC 6741 (a hot and faint star at the centre of a dense, inhomogeneous, equatorial torus merging into a closed, ellipsoidal structure embedded in a large cocoon of almost neutral matter) closely resemble NGC 6565, a compact, recombining PN projected pole-on (Turatto et al. 2002), and show remarkable affinities with NGC 7027, the PNe prototype (Cox et al. 2002; Bains et al. 2003). Very likely, all three objects represent "middle-aged" snapshots of a massive, fast evolving post-AGB star.

\section{Discussion}

In spite of the nebula compactness, we have quite successfully applied tomographic and 3-D analyses to NGC 6741, covered at high spatial and spectral resolution with ESO NTT+EMMI.

NGC 6741 is a young (age $\simeq 1400$ years) PN at a distance of $2000 \mathrm{pc}$; it consists of a high-excitation, almost-prolate ellipsoid $(0.039 \mathrm{pc} \times 0.022 \mathrm{pc} \times 0.019 \mathrm{pc}$; major, intermediate and minor semi-axes, respectively) denser at the equator than at the poles ( $N_{\mathrm{e}}$ peaks at 12000 and $7000 \mathrm{~cm}^{-3}$, respectively), surrounded by a thin skin of low-excitation (the transition zone, corresponding to the present ionization front), and embedded in an extended, spherical (radius $\simeq 0.080 \mathrm{pc}$ ), almost neutral recombining halo containing a large fraction of the nebular mass $\left(M_{\text {halo }} \simeq 0.20 M_{\odot}\right.$ versus $\left.M_{\text {ion }} \simeq 0.06 M_{\odot}\right)$.

The complex ionization structure of NGC 6741 is fully driven by the fast evolution of the central star, a massive $\left(0.66-0.68 M_{\odot}\right)$, hot $\left(\log T_{*} \simeq 5.23 \mathrm{~K}\right)$ and faint $\left(\log L_{*} / L_{\odot} \simeq\right.$ 2.75 ) post-AGB star fading towards the white-dwarf region (after exhaustion of the hydrogen-shell nuclear burning).

The ionized gas of NGC 6741 follows the general expansion law $V_{\text {exp }}\left(\mathrm{km} \mathrm{s}^{-1}\right)=13 \times R^{\prime \prime}$, except the innermost, highestexcitation layers, showing clear evidence of deceleration. This 


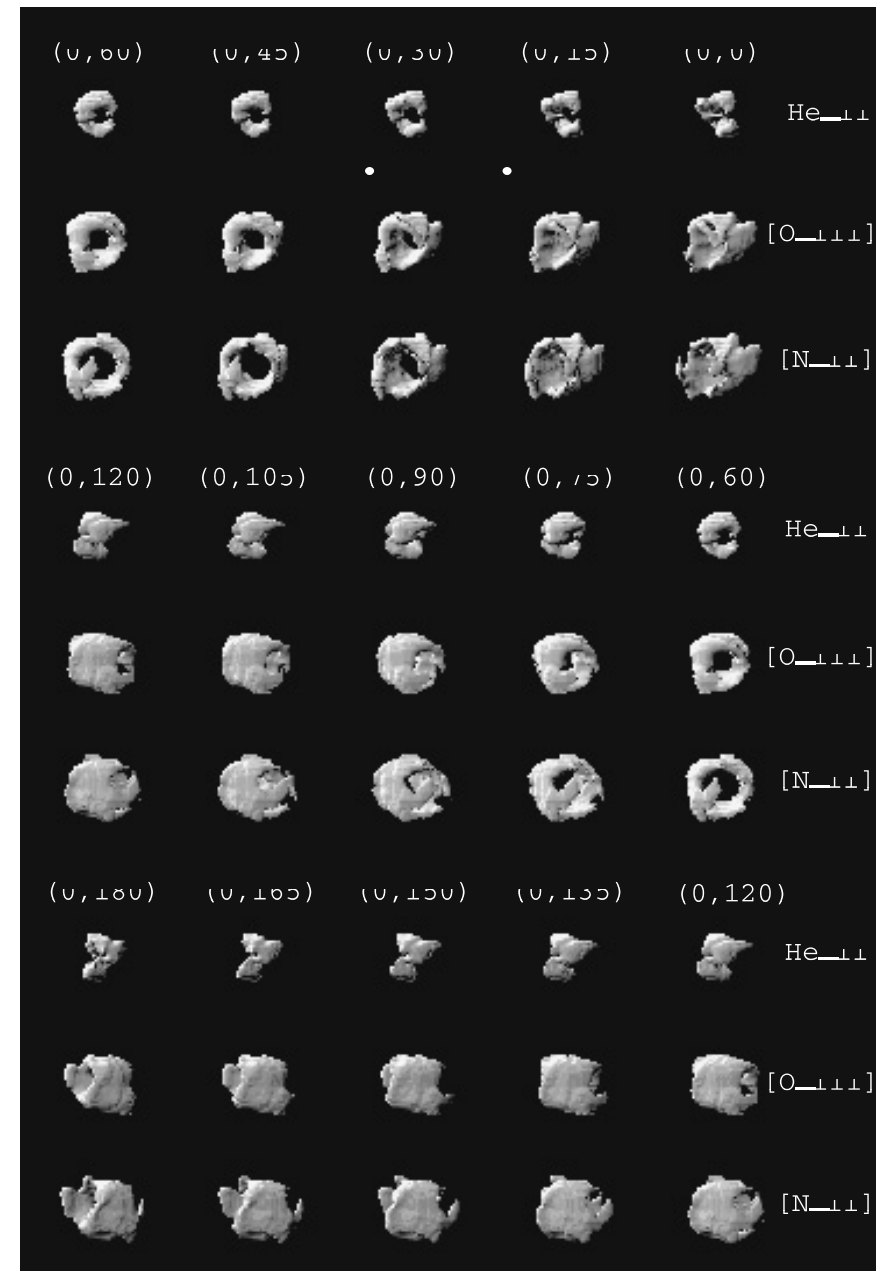

Fig. 16. Stereoscopic structure of NGC 6741 for a rotation around the North-South axis centered on the exciting star. Opaque reconstruction at high flux-cut for $\lambda 4686 \AA$ of He II, $\lambda 5007 \AA$ of [O III], and $\lambda 6584 \AA$ of [N II], as seen from thirteen directions separated by $15^{\circ}$. The line of sight is given by $(\theta, \psi)$, where $\theta$ is the zenith angle and $\psi$ the azimuthal angle. Each horizontal pair represents a "direct" stereoscopic pair (i.e. parallel eyes), and the whole figure provides twelve 3-D views of the nebula in as many directions, covering a straight angle (for details, see Ragazzoni et al. 2001). The $(0,0)$ images represent the rebuilt nebula as seen from Earth (North is up and East to the left). The complete series of nebular movies is shown in the electronic version of the paper (on-line data).

peculiar kinematical behaviour can be qualitatively ascribed to the luminosity drop of the central star (Schönberner et al. 1997; Steffen et al. 1998; Marigo et al. 2001; Perinotto et al. 2004a): in this evolutionary phase the stellar mass-loss quickly decreases, and the falling hot-bubble's pressure no longer balances the pressure of the ionized gas, so that the contact discontinuity and the adjacent, high-excitation nebular layers accelerate inwards.

The argument is poorly known, and deserves more attention, the kinematical properties of the innermost layers being an excellent diagnostic of the star-nebula interaction. A search for more "decelerated" candidates, a high-resolution spectroscopic survey in ionic species at large IP and a detailed comparison with current hydro-dynamical simulations are highly desired.

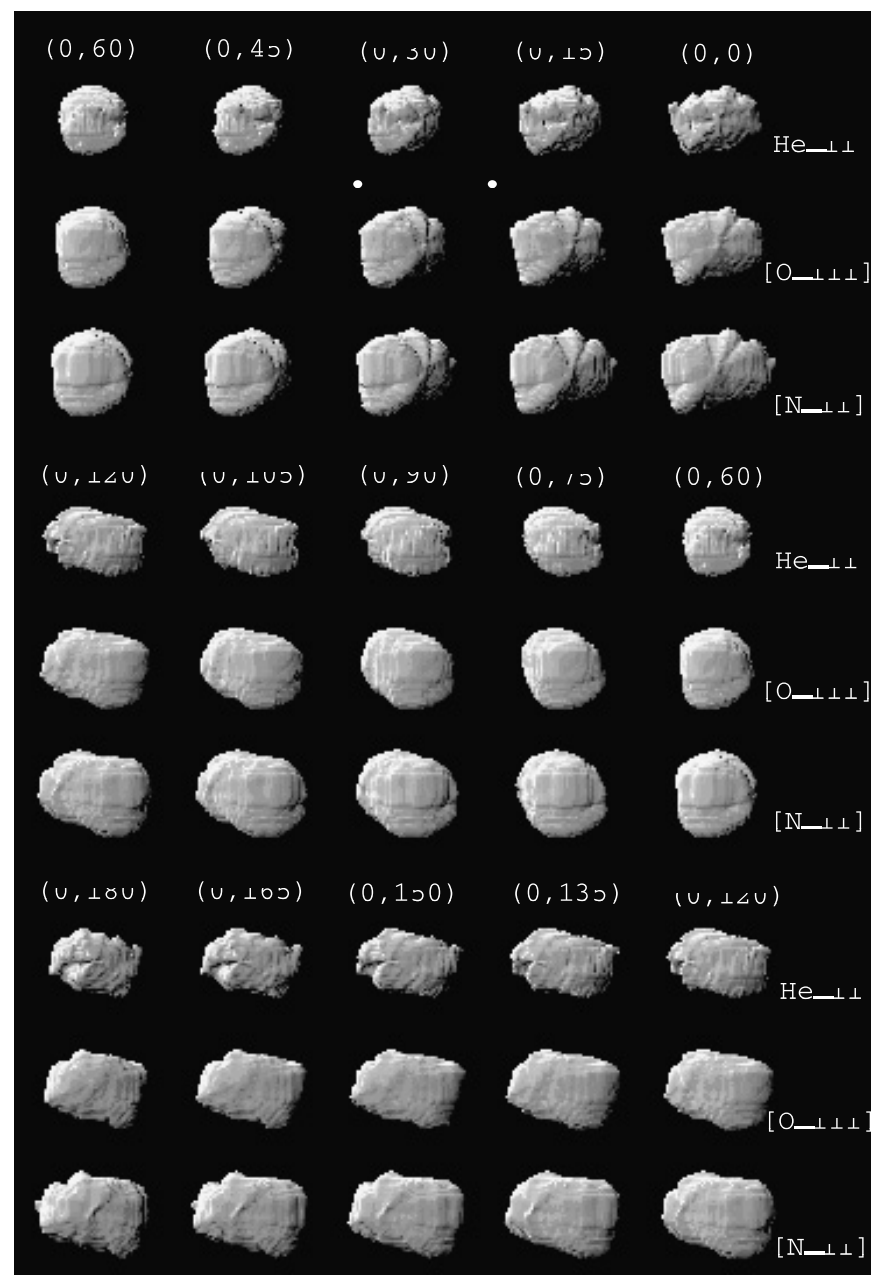

Fig. 17. Same as Fig. 16, but at low flux-cut.

Note that the opposite situation (i.e. acceleration) is expected for the external, recombining layers of NGC 6741, whose de-pressuring gives rise to Rayleigh-Taylor instability at the ionization edge. Although the knotty and filamentary [N II] nebular appearance (Fig. 1, lower panel) qualitatively agrees with this scenario, its confirmation needs very-high spectral resolution $(R \geq 100000)$ echellograms detecting the increase of expansion velocity (and turbulence) at the ionization edge.

A further interesting point concerns the wide radial matter distribution of NGC 6741 (much wider than the $N_{\mathrm{e}}$ profile given in Fig. 13, due to the presence of the dense, almostneutral recombining halo). This indicates a modest contribution of wind interaction to the nebular shaping (it essentially supports the innermost layers, avoiding gas infall), whereas most occurs ionization: the thermal pressure of the ionized gas accelerates the matter outwards and decelerates inwards, creating the density and velocity distributions observed in NGC 6741 (more general comments are given towards the end of this section).

Although the precise radial profile of the matter remains unknown - a large fraction of NGC 6741 being neutral -, the outer radius of the main nebula extends well beyond the present ionization edge. This is supported by the absence of any extended, diffuse, faint and round AGB-halo emission in 


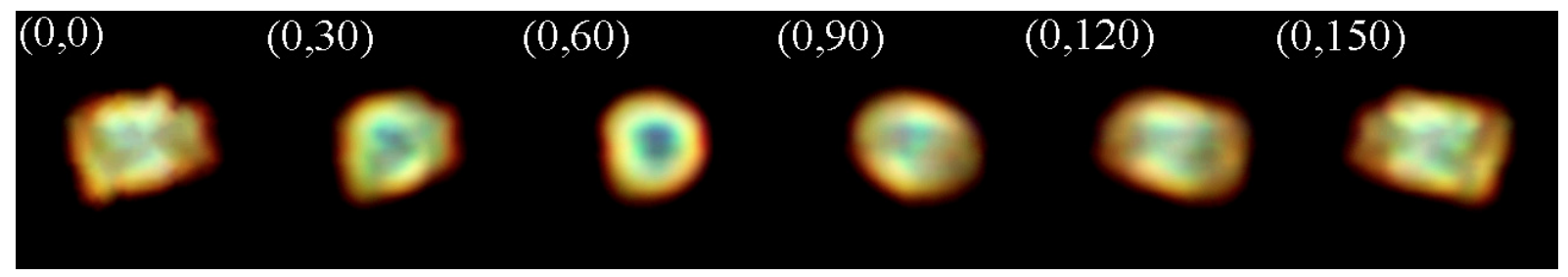

Fig. 18. Optical appearance of NGC 6741 (multi-color in the electronic version of the paper; blue $=$ He II, green $=[\mathrm{O} I I I]$, red $=[\mathrm{N} \mathrm{II}]$ ) for a rotation through the $\mathrm{N}-\mathrm{S}$ axis centered on the exciting star. The left panel, $(0,0)$, corresponds to the re-built nebula as seen from the Earth (North is up and East to the left); projection $(\theta, \psi)=\operatorname{projection}\left(\theta \pm 180^{\circ}, \psi \pm 180^{\circ}\right)$. The complete (multi-color) movie is shown in the electronic version (on-line data).

the Palomar Sky Survey plates, deep imaging by Schwarz et al. (1992), and HST frames. This is confirmed by our spectra: no kinematical signature of an AGB-halo (i.e. external, faint, un-tilted and un-split emission) appears. Since the low-density gas of an AGB-halo (usually $N_{\mathrm{e}}=50$ to $200 \mathrm{~cm}^{-3}$; Chu et al. 1987; Corradi et al. 2003) is little affected by recombination (from Eq. (14), $N_{\mathrm{e}}(0)=50$ to $200 \mathrm{~cm}^{-3}$ implies $N_{\mathrm{e}}(200 \mathrm{yr})=47$ to $\left.162 \mathrm{~cm}^{-3}\right)$, we infer that:

- NGC 6741 never became optically thin to the UV stellar radiation,

- the total nebular mass, $M_{\text {tot }}$, is larger than $0.40 M_{\odot}$.

Let us focus on recombination, which appears as the main characteristic (and peculiarity) of our nebula. As outlined in the previous sections, NGC 6741 is the second PN - of the six so far analysed with the 3-D methodology - in a deep recombination phase (the first being NGC 6565; Turatto et al. 2002). Moreover, according to Benetti et al. (2003), a third nebula, NGC 6818, is at the very beginning of recombination. Such an apparent excess of recombining $\mathrm{PNe}$ is a consequence of our target selection criteria (in particular, high surface brightness and richness of ionic species), favouring massive nebulae powered by fast evolving central stars.

Thanks to the unprecedented accuracy achieved by tomography and 3-D recovery, we have obtained observational evidence supporting the recombination hypothesis for NGC 6741:

(1) nebula much brighter than the star (Sects. 1 and 6);

(2) presence of absorbing knots (Sect. 1);

(3) co-existence of ionic species in a large IP range (Sects. 1, 3 and 7.2);

(4) kinematics of the halo (Sect. 3);

(5) high density of the almost-neutral halo (Sects. 3 and 4);

(6) $c(\mathrm{H} \beta$ ) variable over the nebula (Sect. 4);

(7) high temperature and low luminosity of the central star (Sect. 6);

(8) $T_{\mathrm{Z}} \mathrm{HI}>T_{\mathrm{Z}} \mathrm{He}$ II (Sect. 6).

A minimal, but effective, recombination standard for the whole PN class - in practice, a summary of points (1) and (7) - is given by:

$m\left(V_{*}\right)_{\mathrm{obs}}+\log F(\mathrm{H} \beta)_{\mathrm{obs}}-1.1 \times c(\mathrm{H} \beta)>5.0$,

where $F(\mathrm{H} \beta)_{\text {obs }}$ is in erg $\mathrm{cm}^{-2} \mathrm{~s}^{-1}$.

The Strasbourg-ESO Catalogue of Galactic PNe (Acker et al. 1992) allowed us to select some fifty targets satisfying
Eq. (15). Half of them (e.g. NGC 2440, Hb 4, NGC 6565, NGC 6537, NGC 6620, NGC 6741, NGC 6886, NGC 6881, NGC 7027, Hu 1-2, NGC 6302, and NGC 6563) are compact (sometimes with butterfly-like extentions) at high surface brightness, and represent young recombining PNe ejected and excited by a massive $\left(M_{*} \geq 0.64 M_{\odot}\right)$ fast evolving star beyong the turn-around point in the $\mathrm{H}-\mathrm{R}$ diagram. The remaining candidates are mean-to-low surface brightness, extended (often bipolar) nebulae (e.g. NGC 6445, NGC 6439, A 55, NGC 6772, A 53, NGC 6818, NGC 6894, NGC 7048, NGC 2438, NGC 2818, and IC 4406), corresponding to aged, evolved PNe, optically thick (at least in some directions) to the fading UV radiation of a moderately-massive ( 0.60 to $0.64 M_{\odot}$ ) post-AGB star in the white dwarf cooling sequence.

Further support for the "massive" nature of the central stars of PNe satisfying Eq. (15) is that:

- most targets belong to the morphological class B of Greig (1971, 1972);

- a large fraction consists of type-I PNe (Peimbert 1978; Peimbert \& Torres-Peimbert 1983; Torres-Peimbert \& Peimbert 1983; Peimbert et al. 1995);

- the $\mathrm{H}_{2}$ emission at $2.12 \mu \mathrm{m}$ is commonly detected (Kastner et al. 1996; Natta \& Hollenbach 1998; Bohigas 2001). Note that, although NGC 6741 is not listed among the $\mathrm{H}_{2}$-emitters, a deep search promises fruitful results.

The general rule for recombining $\mathrm{PNe}$ is that the higher the surface brightness of the nebula, the larger the mass of the star.

Thus, recombination represents a not un-common evolutionary phase in $\mathrm{PNe}$; it becomes inescapable for massive nebulae ejected and excited by a massive, fast-evolving post-AGB star (also see Tylenda 1986; Szczerba 1990; Phillips 2000; Marigo et al. 2001; Turatto et al. 2002; Benetti et al. 2003; and Perinotto et al. 2004a).

We end with some general considerations on the kinematics of the whole PN class. The comparative analysis performed in Sect. 3 - rejecting the kinematical results based on spherical symmetry assumption and/or emission profiles of recombination lines integrated along the slit - demonstrates the rarity of real nebulae showing a 'U'-shaped expansion velocity field.

We stress that:

(a) Wilson's law ( $V_{\text {exp }} \propto$ radius) is the general rule for $\mathrm{PNe}$ (Wilson 1950; Weedman 1968; Sabbadin et al. 2004, and references therein); 
(b) at present, only $\mathrm{BD}+30^{\circ} 3639$ and NGC 40 show clear observational evidence of a soft acceleration in the innermost layers (Bryce \& Mellema 1999; Sabbadin et al. 2000a). Both objects: (I) are very-low excitation PNe $(I([\mathrm{O}$ III $] \lambda 5007 \AA)<I(\mathrm{H} \beta))$, (II) exhibit an extremely sharp radial matter profile (shell thickness $\Delta R / R \leq 0.25$ ), and (III) are powered by a "cold" and bright central star of late-WR spectral type. According to Iben et al. (1983), Blöcker (1995, 2001) and Herwig et al. (1999), they represent "born-again" PNe (i.e. the hydrogen-deficient star suffers a late thermal pulse during the motion towards the white-dwarf region. Due to the pulse-induced convection, hydrogen mixes and burns on a convective turn-over time scale);

(c) three more "born-again" candidates - A 30, A 58 and A 78 - are faint, extended PNe presenting a central, complex structure of fast, hydrogen-deficient knots (Jacoby 1979; Pollacco et al. 1992).

This calls for severe constraints on the evolution parameters responsible for PNe shape and shaping (in particular, wind interaction). According to detailed radiation-hydrodynamics simulations (Perinotto et al. 2004a, and references therein), a PN is the result of the synergistic effects of ionization and fast wind on the gas ejected during the superwind phase, generating a typical double-shell structure (inner "rim" + outer "shell") characterized by a "U" or "V"-shaped expansion velocity field. The double-shell structure may only be destroyed either by recombination of the "shell" when the central star fades, or by overtaking of the "shell" by the "rim". In both cases a singleshell configuration emerges, whose expansion velocity field is simple and always increases steadily inwards.

Wind interaction being the main cause of the "V"-shaped expansion profile in double-shell model-PNe, as well of the increasing inwards velocity field in single-shell model-PNe, we infer that all current radiation-hydrodynamics simulations tend to overestimate the dynamical effects of fast stellar wind on the nebular gas (we suspect that post-AGB mass-loss rates adopted by theoretical simulations are systematically too high). Although the same finding arose in the study of NGC 6565 (Turatto et al. 2002), NGC 6818 (Benetti et al. 2003) and NGC 7009 (Sabbadin et al. 2004), a quantitative answer (also including (a) the temporal evolution of mass-loss in the superwind phase, Schönberner et al. 2005, and (b) the binarity and the possible role of magnetic fields, Garcia-Segura \& Lopez 2000; Blackman et al. 2001) will come from a detailed analysis performed on a representative sample of PNe in both hemispheres, covered with ESO NTT+EMMI and TNG+SARG (work in preparation).

\section{Conclusions}

PN observers should avoid use of average line fluxes, integrated spectral profiles, mean electron temperature, overall electron density, and so on. We need detailed, point-to-point flux and velocity measurements over a wide ionization range, combined with a straightforward, versatile method of analysis. To this end, we have applied tomography and 3-D recovery (Sabbadin et al. 2004, and references therein) to long-slit echellograms of the compact, bright PN NGC 6741, covered at nine PA with ESO NTT+EMMI.

We investigated gas kinematics, galactic and circumnebular absorptions, nebular distance, mass and age. We also discussed the stellar properties, nebular physical conditions, ionization structure and photo-ionization model, image deprojection, and multi-color and opaque reconstructions of the nebula.

The results:

- Confirm the unique capacity of tomography and 3-D recovery in extracting the huge amount of physical information stored in high-resolution spectroscopy.

- Stress once more that properly squeezed echellograms represent a peerless tool for deeping the kinematics, physical conditions, ionization structure and evolution of all classes of expanding nebulae (PNe, nova and supernova remnants, shells around Population I Wolf-Rayet stars, nebulae ejected by symbiotic stars, bubbles surrounding early spectral-type Main Sequence stars etc.).

Acknowledgements. We wish to thank the support staff of the NTT (in particular, Olivier Hainaut) for the excellent assistance during the observations.

\section{References}

Acker, A. 1978, A\&AS, 33, 367

Acker, A., Ochsenbein, F., Stenholm, B., et al. 1992, Strasbourg-ESO Catalogue of Galactic Planetary Nebulae (ESO, Garching)

Acker, A., Raytchev, B., Stenholm, B., \& Tylenda, R. 1991, A\&AS, 90, 89

Alexander, J., \& Balick, B. 1997, AJ, 114, 713

Aller, L. H. 1984, Physics of Gaseous Nebulae (Dordrecht: Reidel)

Aller, L. H. 1994, ApJ, 432, 427

Aller, L. H., \& Czyzak, S. J. 1983, ApJS, 51, 211

Aller, L. H., Keyes, C. D., \& Czyzak, S. J. 1985, ApJ, 296,492

Bains, I., Bryce, M., Mellema, G., et al. 2003, MNRAS, 340, 381

Barker, T. 1983, ApJ, 267, 630

Barker, T. 1986, ApJ, 308, 314

Benetti, S., Cappellaro, E., Ragazzoni, R., et al. 2003, A\&A, 400, 161

Berdnikov, L. N., Dambis, A. K., \& Vozyakova, O. V. 2000, A\&AS, 143, 211

Blackman, E. G., Frank, A., \& Welch, C. 2001, ApJ, 546, 288

Blöcker, T. 1995, ApJ, 371, 217

Blöcker, T. 2001, Ap\&SS, 275, 1

Blöcker, T., \& Schönberner, D. 1990, A\&A, 240, L11

Bohigas, J. 2001, Rev. Mex. Astron. Astrofis., 37, 237

Bohlin, R. C., Savage, B. D., \& Drake, J. F. 1978, ApJ, 224, 132

Bryce, M., \& Mellema, G. 1999, MNRAS, 309, 731

Cahn, J. H., Kaler, J. B., \& Stanghellini, L. 1992, A\&AS, 94, 399

Cappellaro, E., Benetti, S., Sabbadin, F., et al. 1994, MNRAS, 267, 871

Chu, Y.-H., Jacoby, G. H., \& Arendt, R. 1987, ApJS, 64, 529

Clegg, R. E. S., Miller, S., Storey, P. J., \& Kisielius, R. 1999, A\&AS, 135,359

Corradi, R. L. M., Schönberner, D., Steffen, M., \& Perinotto, M. 2003, MNRAS, 340, 417

Cox, P., Huggins, P. J., Maillard, J.-P., et al. 2002, A\&A, 384, 603

Curtis, H. D. 1918, Publ. Lick Obs., 13, 55 
De Robertis, M. M., Osterbrock, D. E., \& McKee, C. F. 1985, ApJ, 293, 459

Ercolano, B., Barlow, M. J., Storey, P. J., \& Liu, X.-W. 2003, MNRAS, 340,1136

Feast, M., \& Whitelock, P. A. 1997, MNRAS, 291, 683

Ferland, G. J., Korista, K. T., Verner, D. A., et al. 1998, PASP, 110, 761

Forbes, D. 1985, AJ, 90, 301

Frank, A. 1994, AJ, 107, 261

Garcia-Segura, G., \& Lopez, J. A. 2000, ApJ, 544, 336

Gathier, R., \& Pottasch, S. R. 1988, A\&A, 197, 266

Gesicki, F., Acker, A., \& Zijlstra, A. A. 2003, A\&A, 400, 957

Gesicki, F., \& Zijlstra, A. A. 2003, MNRAS, 338, 347

Greig, W. E. 1971, A\&A, 10, 161

Greig, W. E. 1972, A\&A, 18, 70

Heap, S. R., Corcoran, M. F., Hintzer, P., \& Smith, E. 1989, BAAS, 21, 1199

Herwig, F., Blöcker, T., Langen, N., \& Driebe, T. 1999, A\&A, 349, L5

Hyung, S., \& Aller, L. H. 1997, MNRAS, 292, 71

Iben, I. Jr. 1984, ApJ, 277, 333

Iben, I., Jr., Kaler, J. B., Truran, J. W., \& Renzini, A. 1983, ApJ, 264, 605

Icke, V., Balick, B., \& Frank, A. 1992, A\&A, 253, 224

Jacoby, G. H. 1979, PASP, 91, 754

Kaler, J. B., \& Jacoby, G. H. 1989, ApJ, 345, 871

Kaler, J. B., \& Lutz, J. H. 1985, PASP, 97, 700

Kastner, J. H., Weintraub, D. A., Gatley, I., et al. 1996, ApJ, 462, 777

Keenan, F. P., Aller, L. H., Bell, K. L., et al. 1999, MNRAS, 304, 27

Keenan, F. P., McKenna, F. C., Bell, K. L., et al. 1997, ApJ, 487, 457

Liu, X.-W., Barlow, M. J., Cohen, M., et al. 2001, MNRAS, 323, 343

Lucke, P. B. 1978, A\&A, 64, 367

Lucy, L. B. 1974, AJ, 79, 745

Marigo, P., Girardi, L., Groenewegen, M. A. T., \& Weiss, A. 2001, A\&A, 378, 958

Marten, H., \& Szczerba, R. 1997, A\&A, 325, 1132

Mellema, G. 1997, A\&A, 321, L29

Metzger, M. R., Caldwell, J. A. R., \& Schechter, P. L. 1998, AJ, 115, 635

Natta, A., \& Hollenbach, D. 1998, A\&A, 337, 517

Neiner, C., Acker, A., Gesicki, K., \& Szczerba, R. 2000, A\&A, 358, 321

Osterbrock, D. E. 1989, Astrophysics of Gaseous Nebulae and Active Galactic Nuclei, Mill Valley, CA Univ. Sci.

Palen, S., Balick, B., Hajian, A. R., et al. 2002, AJ, 123, 2666

Peimbert, M. 1978, in Planetary Nebulae, ed. Y. Terzian, IAU Symp., 76, 224

Peimbert, M., \& Torres-Peimbert, S. 1983, in Planetary Nebulae, ed. D. R. Flower, IAU Symp., 103, 233

Peimbert, M., Luridiana, V., \& Torres-Peimbert, S. 1995, RMxAC, 3, 295

Pel, J. W. 1976, A\&AS, 24, 413

Perinotto, M., Schönberner, D., Steffen, M., \& Calonaci, C. 2004a, A\&A, 414, 993

Perinotto, M., Morbidelli, L., \& Scatarzi, A. 2004b, MNRAS, 349, 793
Phillips, J. P. 2000, AJ, 119, 2332

Pollacco, D. L., Lawson, W. A., Clegg, R. E. S., \& Hill, P. W. 1992, MNRAS, 257, 33

Pottasch, S. R. 1981, A\&A, 94, L13

Pottasch, S. R. 1983, in Planetary Nebulae, ed. D. R. Flower, IAU Symp., 103, 391

Pottasch, S. R. 1984, Planetary Nebulae, a Study of Late Stages of Stellar Evolution (Dordrecht: Reidel)

Pottasch, S. R., Beintema, D. A., Bernard Salas, J., \& Feibelman, W. A. 2001, A\&A, 380, 684

Pottasch, S. R., Beintema, D. A., Bernard Salas, J., \& Feibelman, W. A. 2002, RMxAC, 12, 100

Pottasch, S. R., \& Preite-Martinez, A. 1983, A\&A, 126, 31

Ragazzoni, R., Cappellaro, E., Benetti, S., et al. 2001, A\&A, 369, 1088

Reed, D. S., Balick, B., Hajian, A. R., et al. 1999, AJ, 118, 2430

Richardson, W. H. 1972, J. Opt. Soc. Am., 62, 55

Robinson, G. J., Reay, N. K., \& Atherton, P. D. 1982, MNRAS, 199, 649

Sabbadin, F., Benetti, S., Cappellaro, E., \& Turatto, M. 2000b, A\&A, 361,1112

Sabbadin, F., Bianchini, A., Ortolani, S., \& Strafella, F. 1985, MNRAS, 217, 539

Sabbadin, F., Cappellaro, E., Benetti, S., et al. 2000a, A\&A, 355, 688

Sabbadin, F., Cappellaro, E., \& Turatto, M. 1987, A\&A, 182, 305

Sabbadin, F., Turatto, M., Cappellaro, et al. 2004, A\&A, 416, 955

Sagar, R., \& Griffiths, W. K. 1998, MNRAS, 299, 1

Schönberner, D. 1981, A\&A, 103, 119

Schönberner, D. 1983, ApJ, 272, 708

Schönberner, D., Jacob, R., Steffen, M., et al. 2005, A\&A, 431, 963

Schönberner, D., Steffen, M., \& Szczerba, R. 1997, Ap\&SS, 255, 459

Schwarz, H. E., Corradi, R. L. M., \& Melnick, J. 1992, A\&AS, 96, 23

Seaton, M. J. 1968, MNRAS, 139, 129

Seaton, M. J. 1979, MNRAS, 187, 73

Sharpee, B. D., Slanger, T. D., Huestis, D. L., \& Cosby, P. C. 2004, ApJ, 606, 605

Shields, G. A., Aller, L. H., Keyes, C. D., \& Czyzak, S. J. 1981, ApJ, 248,569

Spitzer, L. Jr. 1978, Physical Processes in the Interstellar Medium (New York: John Wiley \& Sons)

Stancil, P. C., Schultz, D. R., Kimura, M., et al. 1999, A\&AS, 140, 225

Stasinska, G. 1989, A\&A, 213, 274

Steffen, M., Szczerba, R., \& Schönberner, D. 1998, A\&A, 337, 149

Szczerba, R. 1990, A\&A, 237, 495

Torres-Peimbert, S., \& Peimbert, M. 1983, PASP, 95, 601

Turatto, M., Cappellaro, E., Ragazzoni, R., et al. 2002, A\&A, 384, 1062

Tylenda, R. 1986, A\&A, 156, 217

Tylenda, R., Acker, A., Gleizes, F., \& Stenholm, B. 1989, A\&AS, 77, 39

Vassiliadis, E., \& Wood, P. R. 1994, ApJS, 92, 125

Weedman, D. W. 1968, ApJ, 153, 49

Wilson, O. C. 1950, ApJ, 111, 279

Wood, P. R., \& Faulkner, D. J. 1986, ApJ, 307, 659 